\title{
Interleukin-18 deteriorates Fabry cardiomyopathy and contributes to the development of left ventricular hypertrophy in Fabry patients with GLA IVS4+919 G>A mutation
}

\author{
Yueh Chien ${ }^{1,7, *}{ }^{,}$Chian-Shiu Chien ${ }^{1,7, *}$, Huai-Chih Chiang ${ }^{1,7, *}$, Wei-Lin Huang ${ }^{1,9}$, \\ Shih-Jie Chou ${ }^{1,7}$, Wei-Chao Chang ${ }^{4}$, Yuh-Lih Chang ${ }^{2,7}$, Hsin-Bang Leu ${ }^{3,8}$, Kuan- \\ Hsuan Chen ${ }^{2,8}$, Kang-Ling Wang ${ }^{3,8}$, Ying-Hsiu Lai ${ }^{8}$, Yung-Yang Liu ${ }^{1,8}$, Kai-Hsi Lu ${ }^{5}$, \\ Hsin-Yang Li ${ }^{1,9}$, Yen-Jen Sung ${ }^{9}$, Yuh-Jyh Jong ${ }^{6}$, Yann-Jang Chen ${ }^{10}$, Chung-Hsuan \\ Chen ${ }^{11,12}$, Wen-Chung $\mathbf{Y u}^{3,13}$ \\ ${ }^{1}$ Department of Medical Research, Taipei Veterans General Hospital, Taipei, Taiwan \\ ${ }^{2}$ Department of Pharmacy, Taipei Veterans General Hospital, Taipei, Taiwan \\ ${ }^{3}$ Division of Cardiology \& Department of Medicine, Taipei Veterans General Hospital, Taipei, Taiwan \\ ${ }^{4}$ Graduate Institute of Cancer Biology and Center for Molecular Medicine, China Medical University and Department of \\ Biotechnology, Asia University, Taichung, Taiwan \\ ${ }^{5}$ Department of Medical Research, Cheng-Hsin Hospital, Taipei, Taiwan \\ ${ }^{6}$ College of Biological Science and Technology, National Chiao Tung University, Hsinchu, Taiwan \\ ${ }^{7}$ Institute of Pharmacology, Taipei, Taiwan \\ ${ }^{8}$ Institute of Clinical Medicine, Taipei, Taiwan \\ ${ }^{9}$ Institute of Anatomy and Cell Biology, Taipei, Taiwan \\ ${ }^{10}$ Department of Life Sciences and Institute of Genome Sciences, Taipei, Taiwan \\ ${ }^{11}$ Genomics Research Center, Academia Sinica, Taipei, Taiwan \\ ${ }^{12}$ Department of Chemistry, National Taiwan University, Taipei, Taiwan \\ ${ }^{13}$ Faculty of Medicine, National Yang-Ming University, Taipei, Taiwan \\ *These authors contributed equally to this work
}

Correspondence to: Wen-Chung Yu, email: wcyu@vghtpe.gov.tw

Keywords: fabry cardiomyopathy, iPSC, enzyme replacement therapy, IL-18

Received: May 11, 2016 Accepted: November 07, $2016 \quad$ Published: November 24, 2016

\section{ABSTRACT}

Rationale: A high incidence of GLA IVS4+919 G>A mutation in patients with Fabry disease of the later-onset cardiac phenotype, has been reported in Taiwan. However, suitable biomarkers or potential therapeutic surrogates for Fabry cardiomyopathy (FC) in such patients under enzyme replacement treatment (ERT) remain unknown.

Objective: Using FC patients carrying IVS4+919 G>A mutation, we constructed an induced pluripotent stem cell (iPSC)-based disease model to investigate the pathogenetic biomarkers and potential therapeutic targets in ERT-treated FC.

Results and methods: The iPSC-differentiated cardiomyocytes derived from FC-patients (FC-iPSC-CMs) carried IVS4+919 G>A mutation recapitulating FC characteristics, including low a-galactosidase A enzyme activity, cellular hypertrophy, and massive globotriaosylceramide accumulation. Microarray analysis revealed that interleukin-18 (IL-18), a pleiotropic cytokine involved in various myocardial diseases, was the most highly upregulated marker in FC-iPSC-CMs. Meanwhile, IL-18 levels were found to be significantly elevated in the culture media of FC-iPSC-CMs and patients' sera. Notably, the serum IL-18 levels were highly paralleled with the progression of left ventricular hypertrophy in Fabry patients receiving ERT. Finally, using FC-iPSC-CMs as in vitro FC model, neutralization of IL-18 with specific antibodies 


\section{combined with ERT synergistically reduced the secretion of IL-18 and the progression of cardiomyocyte hypertrophy in FC-iPSC-CMs. \\ Conclusion: Our data demonstrated that cardiac IL-18 and circulating IL-18 are involved in the pathogenesis of FC and LVH. IL-18 may be a novel marker for evaluating ERT efficacy, and targeting IL-18 might be a potential adjunctive therapy combined with ERT for the treatment of advanced cardiomyopathy in FC patients with IVS4+919 G>A mutation.}

\section{INTRODUCTION}

Fabry disease, resulting from deficiency of $\alpha$-galactosidase A ( $\alpha$-Gal A) enzyme activity, is an lysosomal storage disorder of glycosphingolipids metabolism and leads to accumulation of glycosphingolipids, particularly globotriaosylceramide in many tissues and cell types [1] Fabry disease contains several type of mutation at GLA gene loci, and these mutations might cause different effects at GLA, like transcriptional block, low enzyme activity, or functional lost peptide. Cardiac involvement in Fabry disease usually includes left ventricular hypertrophy (LVH), vascular dysfunction, and conduction abnormalities. In Taiwan, a surprisingly high incidence of GLA IVS4+919 $\mathrm{G}>\mathrm{A}$ mutation of Fabry patients with late-onset cardiac phenotype has been reported. Nevertheless, the natural course and suitable biomarkers for monitoring disease progression remains mostly unclear.

Enzyme replacement therapy (ERT) is currently the only effective therapy to reduce $\mathrm{Gb} 3$ accumulations in Fabry disease. Although it improves cardiac function and left ventricular mass, in patients with late phase Fabry cardiomyopathy (FC), ERT introduced a minor reduction in LVH and have not been able to improve myocardial performance [2]. Although Gb3 and globotriaosylsphingosine (lysoGb3) have high sensitivity and correlate with the severity of LVH in FC [3], recent reports have suggested that $\mathrm{Gb} 3$ and/or lyoGb3 might not be suitable biomarkers for monitoring the long-term therapeutic outcome of ERT and the progression of FC $[4,5]$, including that in Fabry patients carrying IVS4+919 $\mathrm{G}>\mathrm{A}$ mutation [6]. It remains challenging to noninvasively predict the treatment response to ERT. Thus, the identification of pathogenic biomarkers for monitoring patient outcomes in FC, especially in FC patients with myocardial fibrosis, is therefore an urgent need.

Interleukin-18 (IL-18) is a potent pro-hypertrophic inflammatory cytokine and plays a critical role in the pathophysiology of various diseases including myocardial ischemia and myocardial infarction [7]. IL-18 receptor is a heterodimer consisting of a ligand-binding subunit and a signal-transducing subunit. Binding of IL-18 to the ligand-binding subunit recruits the signal-transducing subunit and this activated complex initiates [8] pleiotropic signal transduction events $[9,10]$. This cytokine is upregulated under numerous immune, infectious, and inflammatory conditions (11-13). Administration of IL-18 to normal animals results in increased left ventricular mass and a substantial elevation in myocardial collagen content $[14,15]$. A direct correlation between IL-18 levels and severity of myocardial dysfunction has been observed [16]. Elevated plasma IL-18 levels have been detected in patients with acute coronary syndromes [17]. In addition, IL-18 levels in both the circulation and resident myocardial tissues are increased in patients with heart failure. IL-18 levels inversely correlated with patient prognosis and lower IL-18 levels were observed in surviving patients $[18,19]$. Nevertheless, it remains an open question whether IL-18 plays a role in the pathogenesis underlying FC and LVH.

Induced pluripotent stem cells (iPSCs) provide a feasible platform that can be applied to investigations of tissue repair [20], disease mechanisms [21], drug screening [22] and the regulation of cellular reprogramming [23]. Remarkably, iPSC-derived cardiomyocytes have been considered as a powerful tool for modeling of cardiac diseases [24, 25], assessing of drug cardiotoxicity [26, 27], and investigation of inherited cardiomyopathy $[28,29]$. In addition, patient-specific iPSC-derived cardiomyocytes have been used to evaluate the therapeutic efficacy of gene replacement and small molecule inhibitor on lysosomal storage disorders, including Pompe [30] and Fabry disease [31]. Moreover, transcriptomic screening has been used to identify the gene regulatory network in an iPSC-derived cardiac hypertrophy model [32]. These studies indicated that iPSC-derived cardiomyocytes may represent a powerful tool for the investigation of the therapeutic options and biomarkers for FC. In the present study, we isolated blood mononuclear cells from Fabry patients with IVS4+919 G>A mutation and employed patient-specific iPSC-derived cardiomyocytes to model FC and attempted to evaluate the potential biomarker for FC with IVS4+919 $\mathrm{G}>\mathrm{A}$ mutation. Meanwhile, we also assessed whether neutralization antibody against the identified biomarker could ameliorate the severity of FC in iPSC-derived cardiomyocyte platform. In this present study, we used Affymetrix microarray platform and identified IL-18 as an upregulated factor that may contribute to the pathogenesis of FC and LVH. These findings may help elucidate the underlying mechanisms and identify novel biomarkers for FC and develop novel therapeutic strategies against progressive FC. 


\section{RESULTS}

\section{Establishment of iPSC-derived cardiomyocytes from Fabry patients carrying GLA IVS4+919 G $>$ A mutation}

Fabry disease is a genetic lysosomal storage disorder characterized by glycosphingolipid deposition [35, 36]. The cardiac variant of the IVS4 919G $>$ A mutation accounts for $80 \%$ of Fabry disease incidence in Taiwan, and FC is one of the major highly prevalent Fabry disease-associated morbidities [37]. The most common presentation of FC is left ventricular hypertrophy (LVH) resulting from the progressive intracellular accumulation of Gb3. In the present study, we attempted to use FCderived iPSCs to identify and investigate certain candidate factor that contributed to the cardiomyocyte abnormalities associated with GLA IVS4+919 G>A mutation. Ninetyeight Fabry cohorts with the GLA IVS4+919 G>A intron mutation were recruited for the study at Taipei Veterans General Hospital between 2010 and 2014. The development of FC was diagnosed by cardiologist. Using electroporation to deliver transcription factors OCT4, SOX2, Lin28, KLF4, and p53 shRNA, peripheral blood mononuclear cells (PBMCs) collected from these cohorts were reprogrammed into patient-specific iPSCs (FC-iPSCs). Control iPSC cell-lines (Ctrl-iPSCs) were simultaneously derived from age-matched health subjects (Figure 1A and 1B). No difference was observed in reprogramming efficiency (data not shown), and the expression of embryonic stem cells marker genes (Tra-160 and Tra-1-81) and endogenous pluripotent genes (i.e. OCT4, Nanog, ESG1, DAPP2, DAPP4, REX1, and GDF3) among various patient-derived iPSC lines and Ctrl-iPSCs (Figure 1C and 1D). Using Sanger sequencing, the specific GLA IVS4+919 intron mutation was detected in FC-iPSCs (Figure 1E). Furthermore, these FC-iPSCs also exhibited regular karyotyping, and ability for in vitro tridermal differentiation and teratoma formation (Figure $1 \mathrm{~F}-1 \mathrm{H}$ ).

We next employed conventional cardiomyocyte differentiation protocol and differentiated these FC-iPSCs into cardiomyocytes (FC-iPSC-CMs). Both FC-iPSC$\mathrm{CMs}$ and Ctrl-iPSC-CMs exhibited typical cardiomyocyte morphology and rhythmic contraction at twelve days after cardiac differentiation (Figure 2A). We further used Sanger sequencing and confirmed the GLA IVS4+919 intron mutations in FC-iPSC-CMs, but not Ctrl-iPSC$\mathrm{CMs}$ (Figure 2B). Immunofluorescence indicated that, several distinct myocyte markers, i.e. $\alpha$-actinin, MYL2, MYL7, and cTnT were extensively stained in FC-iPSC$\mathrm{CMs}$ and the Ctrl-iPSC-CMs at 30 days post-induction (Figure 2C). RT-PCR also revealed the upregulation of cardiac maker genes (i.e., HPPA1, NKX2.5, TNNT2, ACTN2, and Myl2) in both FC-iPSC-CMs and CtrliPSC-CMs at 30 and 40 days post-induction (Figure 2D). Notably, no significant discrepancies in the efficiency of cardiac differentiation as well as in the expression levels of these cardiomyocytes markers were observed between these two cells. We further examined whether these FCiPSC-CMs also exhibited FC-specific characteristics after cardiac differentiation. At post-differentiation 40 days in Ctrl and FC-derived cells, $\alpha$-GLA A enzyme activity in the differentiated cardiomyocytes were significantly higher than that in iPSCs. Remarkably, $\alpha$-GLA A enzyme activity were substantially reduced in FC-iPSCs and FCiPSC-CMs, compared with their corresponding Ctrl cells (Figure 2E). Lysosomal abnormalities and $\mathrm{Gb} 3$ accumulation were observed in FC-iPSCs-CMs but not Ctrl-iPSCs-CMs at 40 days post-induction, and the FCiPSC-CMs from all twelve patients displayed similar cardiac hypertrophy and TEM patterns (Figure 2F). In addition, FC-iPSC-CMs exhibited 6-fold higher surface area than that in Ctrl-iPSC-CMs (Figure 2G), revealing the typical phenotypes of cardiomyocyte hypertrophy. Taken together, these FC-iPSC-CMs with IVS4G $>$ A mutation recapitulated several FC-specific phenotypes including lysosomal $\mathrm{Gb} 3$ accumulation, cellular hypertrophy and reduced $\alpha$-GLA A enzyme activity. This FC-iPSC-CM may represent ideal in vitro platform for investigating the pathogenesis of FC and therapeutic strategy for FC.

\section{Identifying IL-18 as an abundant factor in hypertrophic FC-iPSC-CMs}

To identify the most abundant factor differentially expressed between Ctrl-iPSC-CMs and FC-iPSC-CMs, we used Affymetrix microarray platform to identify the differentially expressed genes (Figure 3A). After 40 days of cardiac differentiation, the RNA of FC-iPSC-CMs and Ctrl-iPSC-CMs were extracted, and then subjected to quality verification by Agilent 2100 Bioanalyzer. Following RNA quality verification, the cDNA were synthesized, labeled, and hybridized with Affymetrix human gene chip. The results depicted in the heatmap of Figure 3A showed that a subset of genes is expressed at moderate levels in Ctrl-iPSC-CM with a trend toward higher expression in FC-iPSC-CMs. These genes included the markers and function of heart development likes IL18, EGFR, KCNJ2, PCDH8 and IL-18R1. In addition, the other genes associated with heart development, such as TUBA4A, SLC2A1, CRYBA1 and CRYBA4 also exhibited higher expression levels in FC-iPSC-CMs. (Figure 3A). Among these subsets of genes that were highly expressed in FC-iPSC-CMs, IL-18 was the most upregulated genes expressed in $\mathrm{FC}$-iPSC-CMs, raising the possibility that interleukin-18 (IL-18) may act as a potential biomaker in $\mathrm{FC}$-associated cardiomyopathy.

We next evaluated the regulation of IL-18 expression at mRNA and protein levels in FC-iPSC-CMs during the differentiation course. Quantitative RT-PCR revealed that IL-18 and its receptor (IL-18R) were both upregulated, whereas IL-18 binding protein (IL-18BP) 
was downregualted, in a time-dependent manner along the differentiation course (Figure 3B). Western blot revealed that IL-18 was initially increased at post-induction Day 30 , and was further upregulated time-dependently and reached plateau at post-induction Day 50 (Figure 3C). By measuring the cell surface area, a severe cardiomyocyte hypertrophy was detected at post-induction 40 day in FCiPSC-CMs (Figure 3D). In addition, immunofluorescence also indicated that IL-18 protein was significantly higher in FC-iPSC-CMs than that in Ctrl-iPSC-CMs at postinduction 40 day (Figure 3E and 3F). Furthermore, ELISA indicated that IL-18 was secreted into the conditioned media and elevated in the manner identical to IL-18 protein during the cardiac induction (Figure $3 \mathrm{G}$ ). These findings revealed that IL-18 is a FC-specific factors upregulated during the differentiation process in FC-iPSC-CMs.

\section{IL-18 as a candidate FC biomarker in FC patients with IVS4G $>$ A mutation}

In order to determine the key molecules that involved in the FC, the Ingenuity Pathway Analysis (IPA) was used to address the interaction network of the differentially expressed genes in FC-iPSC-CMs. Mapping these genes to the network of cardiac hypertrophy revealed the vital role of IL-18 that participate in FC (Figure 4A). IL-18 was illustrated to interact with the cytokines/chemokines
(IL6, and CXCL12), cytosolic signaling molecules (NOS2, PI3K/Akt, MAPK cascade, and AMPK), and redox transcription factors (AP1, and NF- $\kappa \mathrm{B})$. Moreover, the IL18-signaling downstream effectors, i.e. ATF3, CTNNB1, STAT3, SMAD7, GATA4, focal adhesion kinase, and MMP1 have also been illustrated by IPA. All of these molecules play the important role in the progression of hypertrophic cardiomyopathy. Overall, the bioinformatics approach using IPA markedly pointed out that IL-18 acts as a critical initiator in the network of cardiac hypertrophy, suggesting the potential involvement of the secreted IL-18 on FC progression.

To further elucidate the role of IL-18, we also assessed whether IL-18 is also elevated in the circulation in clinical samples from Fabry patients. First, we examined the circulatory IL-18 levels in 25 Fabry patients who carried IVS4+919 G>A mutation and have not developed left ventricular hypertrophy (LVH). There was no significant difference in transthoracic echocardiography parameters including left ventricular mass (LVM) and left ventricular mass index (LVMI) (all transthoracic echocardiography parameters $P>0.05$, Figure 4B and $4 \mathrm{C})$. Notably, these patients exhibited slightly higher elevation in conventional FC-specific biomarker lysoGb3, as well as IL-18 (Figure 4D and 4E).

Next, we recruited 30 Fabry patients with IVS4+919 G>A mutation and have never received
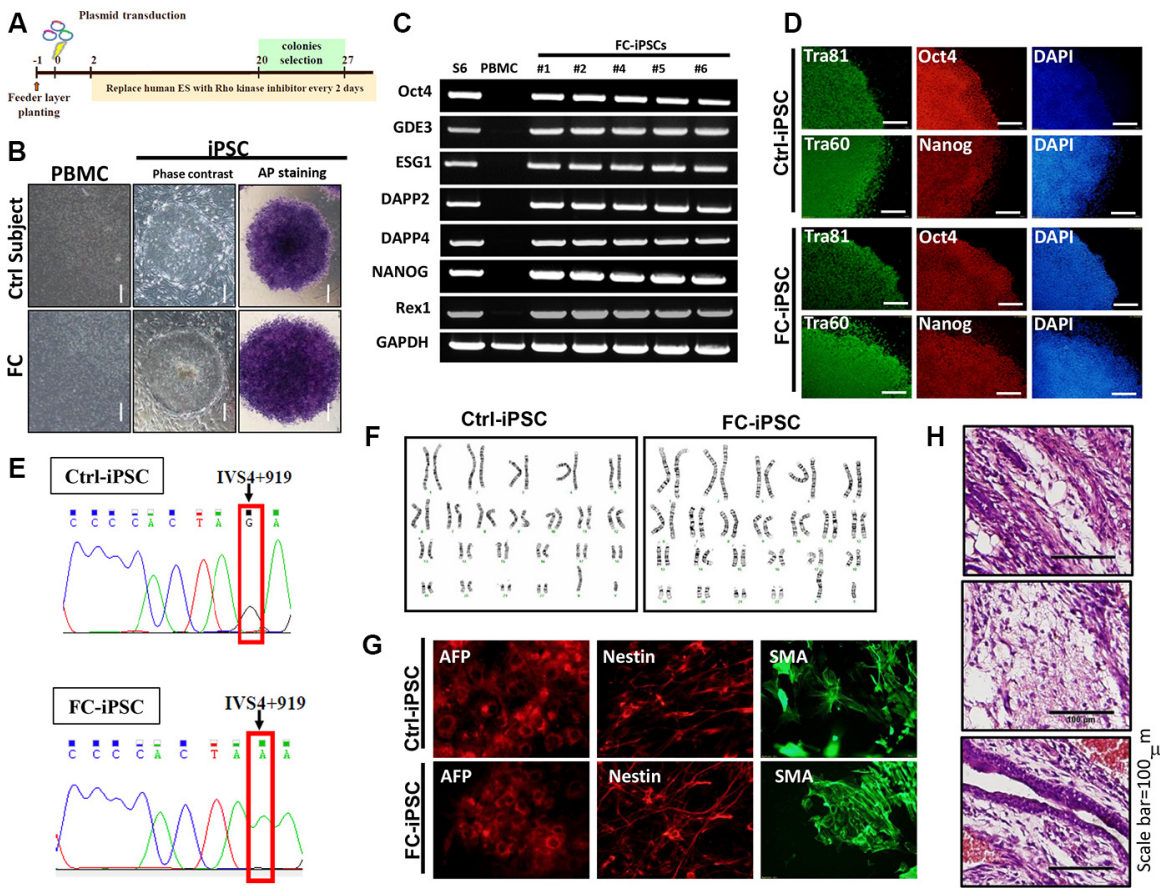

Figure 1: Generation of FC-iPSCs from Fabry patients carrying GLA IVS4+919 G>A mutation. (A) Protocol for iPSC generation from peripheral blood mononuclear cells (PBMCs) collected from the Fabry cohorts carrying GLA IVS4+919 G>A mutation. (B) Phase-contrast photomicrograph and ALP activity in FC-iPSCs. (C) Reverse-transcriptase polymerase chain reaction (RT-PCR) results showed that FC-iPSC clones expressed the endogenous pluripotency markers such as OCT4, Nanog, ESG1, DAPP2, DAPP4, REX1, and GDF3. (D) Immunofluorescence results indicated that the FC-iPSC and Ctrl-iPSC colonies both showed strong expression of the hESC markers, including Oct4, Nanog, Tra-1-60 and Tra-1-81. (E) Sanger sequencing revealed the specific GLA IVS4+919 intron mutation in FC-iPSCs. (F) Karyotyping and the abilities for $(\mathbf{G})$ teratoma formation and $(\mathbf{H})$ in vitro tridermal differentiation. 
enzyme replacement therapy and have developed Fabry cardiomyopathy (FC), and further compared their transthoracic echocardiography and serum levels of LysoGb3 and IL-18, with those in age-matched control subjects or hypertensive patients with LVH only (Table 1). The severity of LVH in these patients was diagnosed by transthoracic echocardiography. Comparing with those parameters in age-matched control subjects, both Fabry patients with IVS4+919 G>A mutation and patients with LVH exhibited high magnitudes of LVM and LVMI (all transthoracic echocardiography parameters $P<0.001$, Figure 5A and 5B). In addition to all echocardiography parameters, the serum lysoGb3 concentration was higher in Fabry patients with IVS4+919 G>A mutation, but not in patients with LVH alone, comparing with the controls (Figure 5C). Remarkably, the serum IL-18 concentrations were slightly increased in patients with LVH alone, and were even much higher in Fabry patients carried IVS4+919 G>A mutation and have developed LVH (Figure 5C and 5D). Histological examination and transmission electron microscopic examination were used to validate the microscopic characteristics in these Fabry patients who have developed FC. A hematoxylin and eosin examination of the biopsied myocardium sample revealed cardiomyocyte hypertrophy and disorganization with large perinuclear and sarcoplasmic vacuoles (Figure 5E). Toluidine blue staining of the biopsied myocardium indicated the accumulation of glycosphingolipids (Figure 5F). Transmission electron microscopic examination of the myocardium revealed the formation of lamellar bodies (zebra bodies) which represent lysosomes containing glycolipids (Figure 5G and 5H). Collectively, these in vitro and clinical findings (Figures 3-5) indicated that IL-18 is highly elevated and associated with Fabry patients with FC or LVH and could be used as a diagnostic marker in Fabry patients who have developed LVH.
A
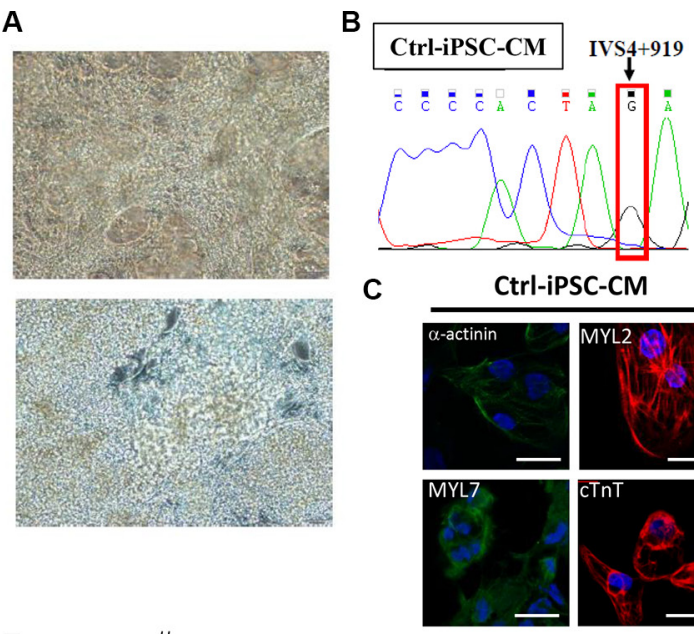

E

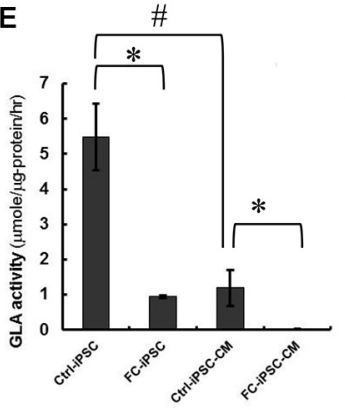

$\mathbf{F}$

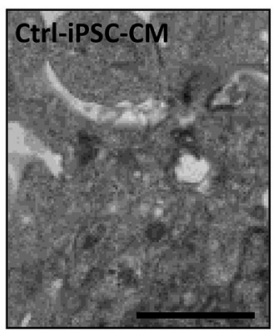

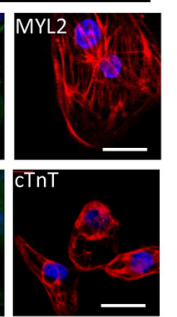

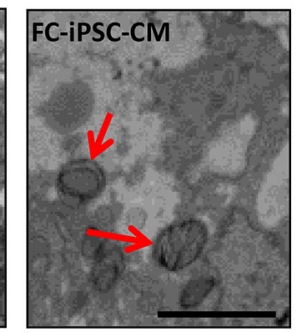

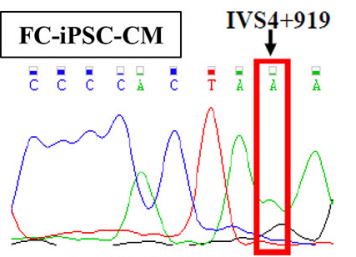

FC-iPSC-CM
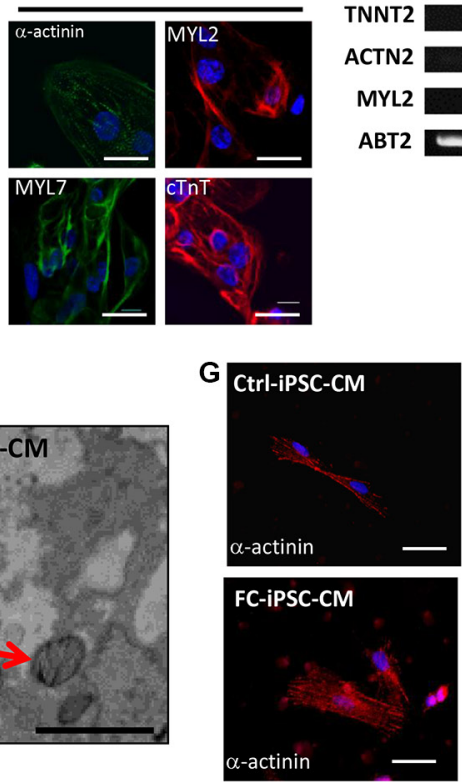

D

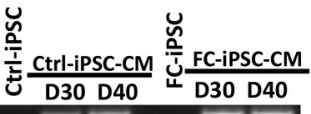

HPPA1

NKX2.5

TNNT2

ACTN2

MYL2

ABT2
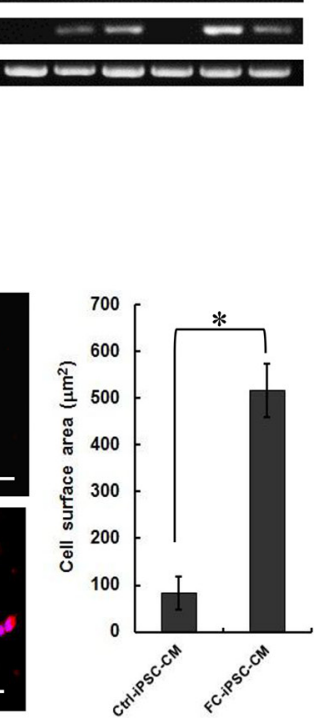

Figure 2: Establishment of FC-iPSC-CMs that recapitulated several FC-specific characteristics. (A) FC-iPSC-CMs exhibited typical cardiomyocyte morphology and rhythmic contraction at twelve days after cardiac differentiation. (B) Sanger sequencing revealed the specific GLA IVS4+919 intron mutation in FC-iPSC-CMs, but not in Ctrl-iPSC-CMs. (C) Immunofluorescence results indicated that FC-iPSC-CMs and Ctrl-iPSC-CMs both showed strong expression of myocyte markers, i. e. $\alpha$-actinin, MYL2, MYL7, and cTnT. (D) Reverse-transcriptase polymerase chain reaction (RT-PCR) results showed upregulation of cardiac maker genes (i.e., HPPA1, NKX2.5, TNNT2, ACTN2, and Myl2) in both FC-iPSC-CMs and Ctrl-iPSC-CMs at 30 and 40 days post-induction. (E) Measurements of $\alpha$-GLA A enzyme activity in Ctrl-iPSCs, FC-iPSCs, Ctrl-iPSC-CMs and FC-iPSC-CMs. (F) TEM examination of FC-iPSC-CMs showed the morphological abnormalities (organelle and/or cytoplasm loss) and Gb3 accumulation (appearing within enlarged secondary lysosomes as lamellated membrane structures called zebra bodies) $(\mathrm{TEM} \times 8,000)$. (G) Measurement of cell surface area indicated the cardiomyocyte hypertrophy in FC-iPSC-CMs. 
Table 1: Patient information

\begin{tabular}{|c|c|c|c|}
\hline & $\begin{array}{l}\text { Healthy control } \\
\qquad N=\mathbf{3 0}\end{array}$ & $\begin{array}{c}\text { LVH } \\
N=22\end{array}$ & $\begin{array}{c}\text { Fabry } \\
\text { cardiomyopathy }(\text { FC) } N=25\end{array}$ \\
\hline Age, yrs & $59.1 \pm 4.5$ & $55.2 \pm 6.2$ & $58.4 \pm 5.8$ \\
\hline Women, no. (\%) & $1(3 \%)$ & $2(9 \%)$ & $1(4 \%)$ \\
\hline High, cm & $155 \pm 5.3$ & $157 \pm 4.2$ & $156 \pm 3.6$ \\
\hline Weight, $\mathrm{kg}$ & $56 \pm 5.5$ & $57 \pm 5.8$ & $56 \pm 3.9$ \\
\hline Body mass index, $\mathrm{kg} / \mathrm{m} 2$ & $26.1 \pm 2.2$ & $25.4 \pm 2.4$ & $25.8 \pm 2.9$ \\
\hline MSSI cardiovascular score & - & - & $12.1 \pm 2.2$ \\
\hline Hypertension, no. (\%) & - & $12(54 \%)$ & $12(48 \%)$ \\
\hline LVH, no. (\%) & - & $22(100 \%)$ & $25(100 \%)$ \\
\hline Ccr $<60 \mathrm{ml} / \mathrm{min}$, no. $(\%)$ & - & $22(100 \%)$ & $25(100 \%)$ \\
\hline ACE/ARB inhibitors use & - & $22(100 \%)$ & $20(80 \%)$ \\
\hline Left ventricular mass, $\mathrm{g}$ & $169 \pm 7.3$ & $232.3 \pm 20.5$ & $260.4 \pm 23.1$ \\
\hline Left ventricular mass index & $50 \pm 0.6$ & $65.1 \pm 2.5$ & $71.5 \pm 3.5$ \\
\hline Left ventricular ejection fraction, $\%$ & $70 \%$ & $65 \%$ & $69 \%$ \\
\hline NT-pro-BNP & - & $984 \pm 10.5$ & $703 \pm 12.5$ \\
\hline LysoGb3, nM & $0.3 \pm 0.01$ & $0.3 \pm 0.03$ & $3.5 \pm 0.4$ \\
\hline IL18, ng/mL & $0.8 \pm 0.01$ & $0.5 \pm 0.04$ & $7.5 \pm 0.2$ \\
\hline
\end{tabular}

Data are mean \pm SD. MSSI, Mainz severity score index; Ccr: Creatinine Clearance.

\section{Enzyme replacement therapy decreased left ventricular hypertrophy and ameliorated serum levels of lysoGb3 and IL-18 in patients with fabry cardiomyopathy}

Because our data indicated that the serum levels of IL-18 were significantly upregulated in patients diagnosed as FC, we evaluated whether IL-18 could serve as circulating biomarkers that mirror ERT efficacy and disease progression in FC. A total of 30 Fabry patients carried IVS4+919 G>A mutation and have developed LVH and received ERT were enrolled in this study, and LVH reduction was used as a surrogate endpoint to compare several parameters before and after ERT. The severity of $\mathrm{LVH}$, as indicated by echocardiography parameters and lyoGb3 concentration, was reduced after ERT $((\Delta=-38.7$ $(P=0.04),-9.7(P=0.01)$, and $-2.1(P=0.01)$, for LVM, LVMI, and lyoGb3, respectively) along with the reduction of IL-18 $(\Delta=-4.5(P<0.01))$ (Figure 6A). We previously have reported that pro-inflammatory cytokines, particularly IL-6 and MCP-1, were elevated in patients with FC and carrying GLA IVS4+919 G>A mutation [38]. In addition, $\mathrm{N}$-terminal pro-brain natriuretic peptide is a marker of left ventricular hypertrophy in hypertrophic cardiomyopathy [39]. Subsequently, we evaluated the correlation between the changes in nt-ProBNP, lysoGb3, IL-6, IL-18 and the changes in LVMI. The correlations between LVMI and these serum biomarkers were all positive (beta $=0.55$ $(P<0.05), 0.68(P<0.05), 0.74(P<0.05)$, and 0.67
$(P<0.05)$ for nt-ProBNP, IL-6, lyoGb3 and IL-18, respectively) (Figure 6B). The beta values for IL-18 and IL-6 were equivalent, whereas considering the critical roles of IL-18 in heart diseases and cardiac hypertrophy, IL-18 may represent a better biomarker that can reflect the development of FC in patients with IVS4+919 G>A mutation. We further assessed the correlation between the changes in IL-18 or LysoGb3 and the changes in LVMI in 5 of 30 patients with LVH progression after ERT. Only IL-18 was positively correlated with LVMI (beta $=0.51$ $(P<0.05)$, respectively), whereas lyoGb3 had no correlation with LVMI (beta $=5 \times 10^{-5}$ ) (Figure 6C). This discordance between reduced lysoGb3 and the progression of LVH signifies its limitation for prognostic implications. Meanwhile, the levels of IL-18 were elevated and highly paralleled the progression of LVH even under standard ERT (Figure 7A). Finally, immunohistochemical results further confirmed that the expression levels of IL-18 were significantly increased in the biopsy samples of FC patients with poor responses to ERT compared with those who responded well to ERT (Figure 7B). Collectively, our clinical findings revealed that conventional marker lysoGb3 may not be a reliable marker for Fabry-associated ventricular hypertrophy, particularly in patients with LVH progression after ERT. Alternatively, serum IL-18 level may be an ideal biomarker for revealing Fabry-associated cardiac manifestations with high sensitivity, specifically for monitoring progressive $\mathrm{LVH}$ in $\mathrm{FC}$ patients undergoing ERT. 


\section{Neutralization of IL-18 enhances ERT efficacy on cardiomyocyte hypertrophy in FC-iPSC-CMs}

Disease-specific iPSCs provide a high-throughput platform for personalized drug screening and clinicaloriented therapeutic development [40]. Therefore, we evaluated the treatment response of FC-iPSC-CMs to ERT. Six FC-iPSC-CM clones that consistently exhibited Fabry-associated characteristics, including cardiomyocyte hypertrophy, decreased GLA expression/activity, and lysosomal abnormalities at 40-60 days post-induction, were allocated for subsequent experiments.

First, we tested whether ERT could restore in vitro GLA activity in FC-iPSC-CMs at 30 days post-induction. The administration of alpha-galactosidase A (Replagal $5 \mu \mathrm{g} / \mathrm{ml}$ for 4 days) elicited high GLA activity in ESC- derived cardiomyocytes, Ctrl-iPSC-CMs and FC-iPSCCMs. Notably FC-iPSC-CMs exhibited a significantly lower GLA activity than the control cells after alphagalactosidase A administration (Figure 8A).

Second, we tested the treatment response of alpha-galactosidase A at early onset on cardiomyocyte hypertrophy in FC-iPSC-CMs. To ensure the restoration of GLA activity, medium containing alpha-galactosidase A was refreshed every two days. There was a significant decrease in cardiomyocyte size on day 35 in the FC-iPSC$\mathrm{CMs}$ that were treated from post-induction day 0 to day 60 (Figure 8B). At day 60, the cell size of the treated FCiPSC-CMs was approximately $31 \%$ smaller than that of the untreated FC-iPSC-CMs (Figure 8B). LysoGb3 release was also reduced by alpha-galactosidase $\mathrm{A}$, which is a known effect of this enzyme, during the differentiation
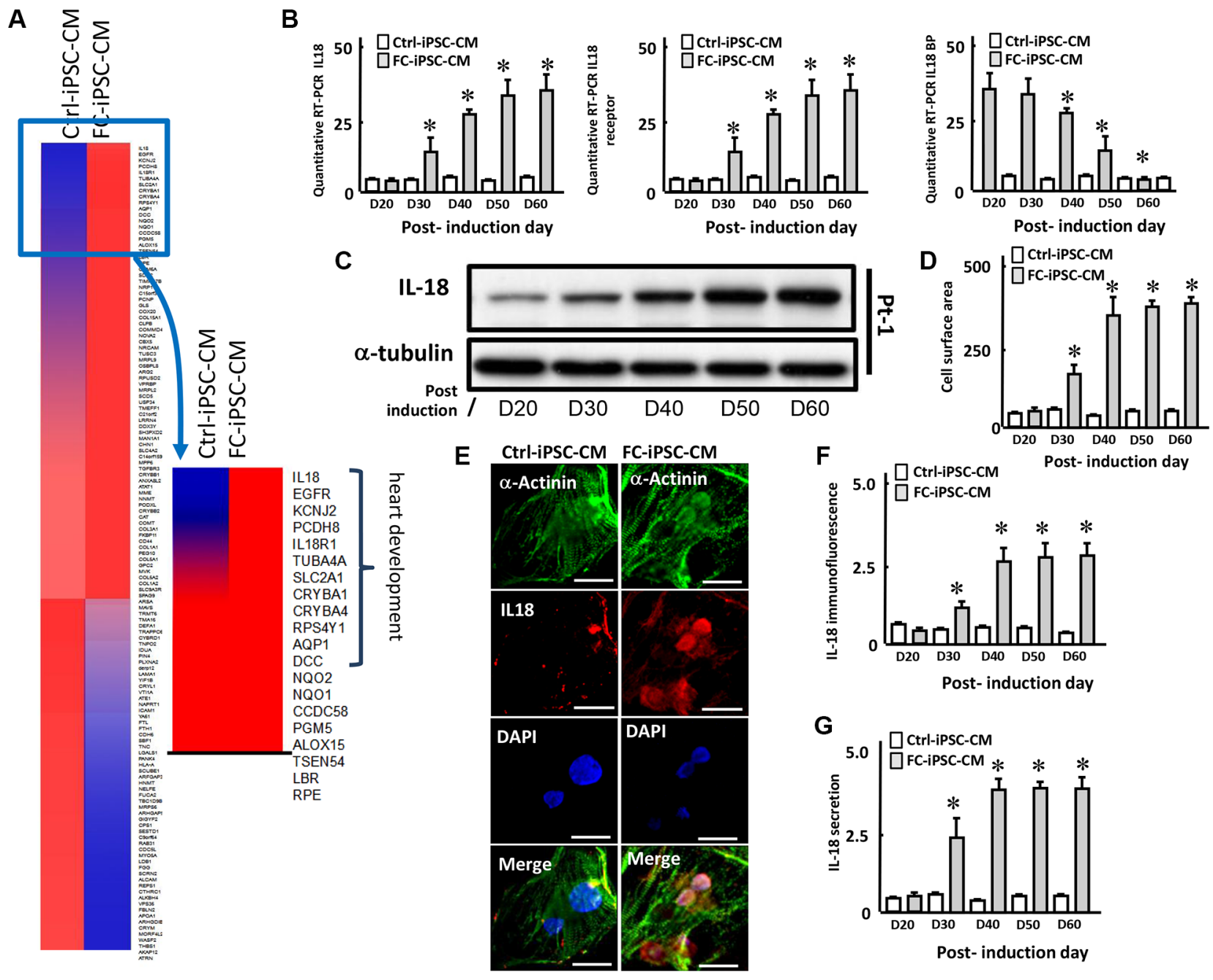

Figure 3: L-18 as a highly upregulated factor in FC-iPSC-CMs. (A) Heatmap depicting fold change of expression in the different genes between FC-iPSC-CM and iPSC-CM. Most different genes between two cells expression are grouped in heart development. (B) Quantitative RT-PCR revealed the expression of IL-18, IL-18 receptor (IL-18R) and IL-18 binding protein (IL-18BP) along the differentiation course. (C) Western blot showed the expression of IL-18 during cardiomyocyte differentiation. (D) Measurement of cell surface area in FC-iPSC-CMs during the cardiomyocyte differentiation. (E) Immunofluorescence result and (F) the quantification indicated the IL-18 upregulation in FC-iPSC-CMs than that in Ctrl-iPSC-CMs at post-induction 40 day. (G) ELISA showed the secretion of IL-18 into the conditioned media during the cardiac induction. 
course of the FC-iPSC-CMs (Figure 8C). These data showed that ERT effectively prevented the development of cardiomyocyte hypertrophy in early onset FC.

Third, we attempted to test the efficacy of ERT in FC-iPSC-CMs with severe cardiomyocyte hypertrophy at post-induction day 40 (Figure $8 \mathrm{D}$ and $8 \mathrm{E}$ ). Alphagalactosidase A treatment from post-induction day 40 to day 60 resulted in a negligible inhibitory effect on cardiomyocyte size (Figure 8D), despite reduced levels of lysoGb3 secretion (Figure 8E). Meanwhile, we examined whether IL-18 could serve as therapeutic targets at this stage, and we also tested the treatment efficacy of the neutralization of IL-18 in combination with ERT (Figure 8F and 8G). Remarkably, the addition of an IL-18 neutralization antibody (IL-18 nAb) potently enhanced the efficacy of alpha-galactosidase A and reduced the cardiomyocyte size (Figure 8F), accompanied by a decrease in the production of lysoGb3 (Figure 8G). Immunofluorescence further indicated that the cardiacspecific marker $\alpha$-actinin was unaffected, whereas fewer FC-iPSC-CMs expressed IL-18 when receiving the combination treatment than the cells receiving ERT only (Figure $8 \mathrm{H}$ ). As detected by ELISA, the secretion of IL-18 (Figure 8I), were generally unaffected by ERT alone but were moderately attenuated by IL-18 nAb (Figure 8I, left). Similarly, the addition of IL-18 nAb enhanced the effects of ERT and exhibited a synergistic efficacy that potently suppressed IL-18 (Figure 8I, left). The release of lysoGb3 was consistently reduced by administration of Alphagalactosidase A but not IL-18 nAb (Figure 8I right).

Furthermore, we chose several cardiac hypertrophyassociated genes (i.e. ANF, ACTC1, MYL2, and MYL7) that are upregulated in FC-iPSC-CMs (unpublished data) and examined the efficacy of alpha-galactosidase $\mathrm{A}$ (Replagal), IL-18 nAb, or alpha-galactosidase A plus IL$18 \mathrm{nAb}$ on the expression of these hypertrophy-associated genes. As detected by quantitative RT-PCR, alphagalactosidase $\mathrm{A}$ alone did not significantly affect these genes. IL-18 nAb alone showed a moderate suppressive effect and the combination of alpha-galactosidase A plus IL-18 nAb synergistically suppressed these selected hypertrophy-associated genes (Supplemental Figure S1). These data indicated the ineffectiveness of ERT drug on the expression of cardiac hypertrophy-associated genes as well as cardiomyocyte hypertrophy. These in vitro findings using FC-iPSC-CMs were consistent with clinical observations by Kampmann et al. and our previous study $[41,42]$.
A

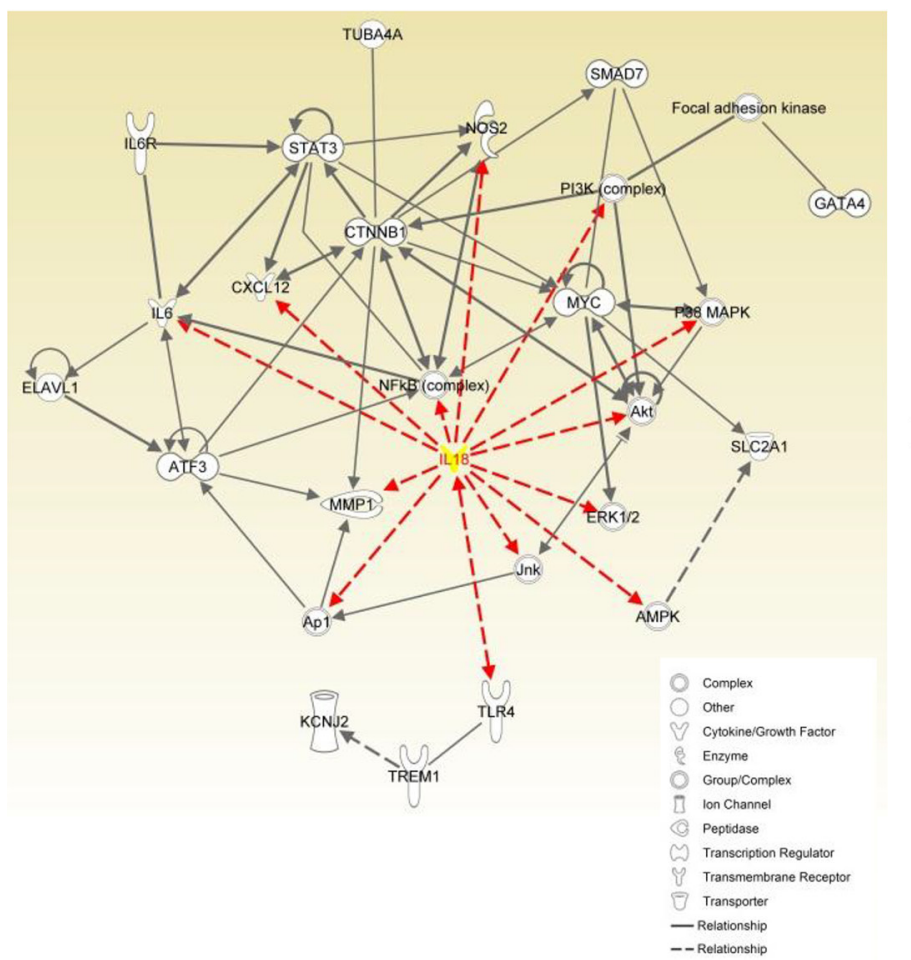

B

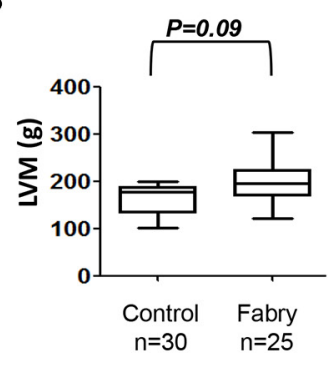

D

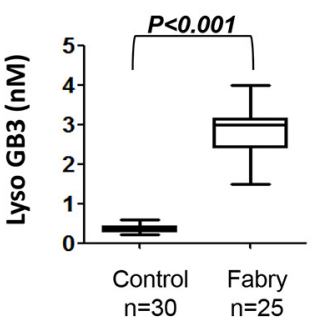

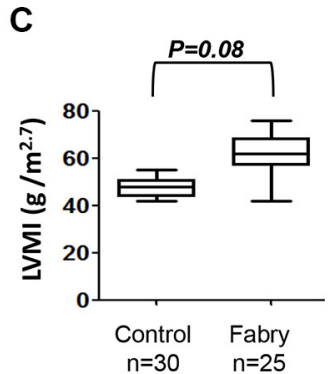

E

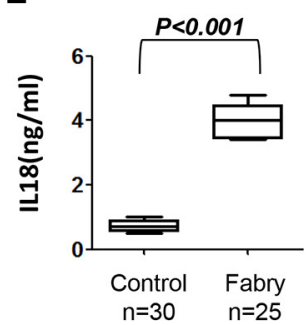

Figure 4: Upregulation of IL-18 in Fabry patients carrying GLA IVS4+919 intron mutation. (A) The associated interaction network was defined by Ingenuity Pathway Analysis (IPA) for IL-18 and the cardiac hypertrophy related genes. The IL-18 was labeled as yellow and the interaction linage between IL-18 and other molecules was plotted as red-line. (B, C) Transthoracic echocardiography parameters and the serum levels of (D) lysoGb3, and (E) IL-18 in 25 FC patients carrying GLA IVS4+919 intron mutation and without the development of LVH. 


\section{Low GLA expression might not be the predominant factor that stimulates the IL-18 secretion}

In addition to the enrolled Fabry patients with the IVS4+919 G>A mutation predominantly found in Taiwan, we have also recruited a small population of Fabry cohorts with the classic type mutation. To examine the IL-18 expression pattern, we also collected the peripheral mononuclear blood cells from Fabry patients with classic type mutation and reprogrammed them into FC-iPSC (classic type). Subsequently, we also differentiated them into FC-iPSC-CMs (classic type), and further compared the expression and secretion patterns of IL-18 in FC-iPSC$\mathrm{CMs}$ (classic type) with that in FC-iPSC-CMs (IVS4+919 $\mathrm{G}>\mathrm{A}$ ) and Ctrl-iPSC-CMs. A moderate IL-18 upregulation as well as its secretion were observed in FC-iPSC-CMs (classic type). These data related to classic type mutation are showed in the Figure 9A.

To assess the consequence of GLA knockdown on IL-18 secretion, we compared the IL-18 secretion and GLA activity in FC-iPSC-CMs receiving shRNA against GLA (shGLA) or shLuc at different time points after cardiac induction (D30, and D60). Knockdown of GLA by ShGLA led to expected reduction in GLA activity in FC-
iPSC-CMs at post-induction 30 days, compared with the same cells receiving ShLuc or those without any treatment. Nevertheless, no difference in GLA activity was detected in FC-iPSC-CMs among untreated and shGLA-treated cells at post-induction 60 days. Remarkably, a significant increase in IL-18 secretion was observed in FC-iPSCCMs with GLA knockdown at post-induction day 30, whereas high levels of IL-18 secretion were detected in FC-iPSC-CMs among all groups with any given treatment (Figure 9B). Our in vitro data using Fabry patient-derived iPSC platform indicated that low GLA expression only moderately elicited IL-18 secretion, suggesting that low GLA expression might not be the predominant factor that stimulates the IL-18 secretion.

\section{DISCUSSION}

Gal A enzyme deficiency in Fabry patients results in progressive accumulation of cellular $\mathrm{Gb} 3$ in a variety of cell types, including the walls of small blood vessels, tubular epithelial cells, nerves, dorsal root ganglia, renal glomerular and cardiomyocytes [43, 44]. The GLA IVS4+919 G>A mutation has been revealed as a high incidence of GLA mutation ( $\sim 1$ in 1,500-1,600 males) of Fabry patients in Taiwan [45]. Moreover, this mutation
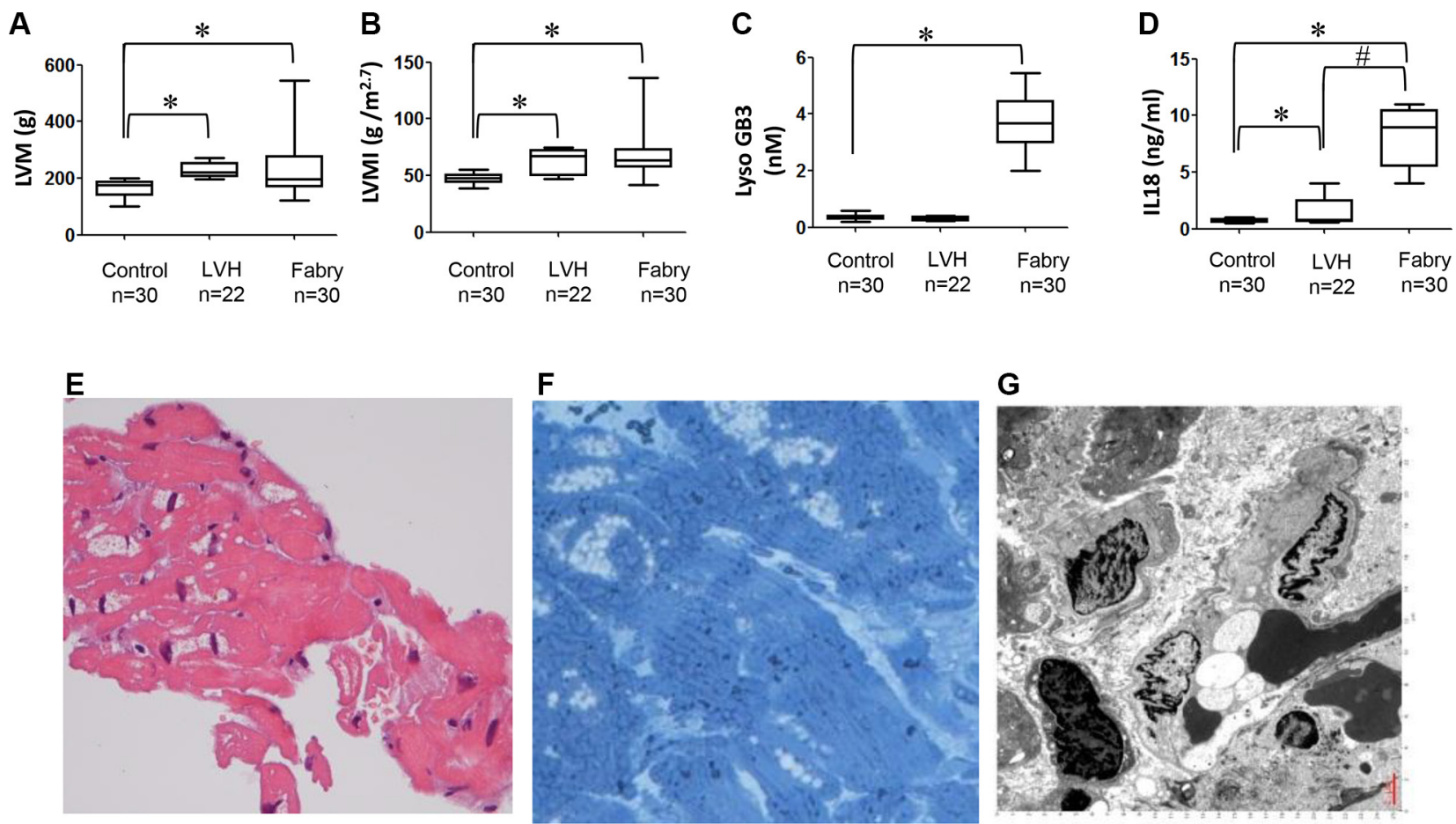

Figure 5: Comparison of secretory IL-18, LysoGb3 and transthoracic parameters among FC patients with LVH, hypertensive patients with LVH only and control subjects. (A-D) Transthoracic echocardiography parameters and the serum levels of lysoGb3, IL-18 in 30 FC patients with LVH, 22 hypertensive patients with LVH only, and 30 control subjects. (E) Histologic examination (H\&E) staining of the myocardium showed markedly hypertrophic and disorganized myocytes with large perinuclear and sarcoplasmic vacuoles (upper right). (F) Toluidine blue staining of the myocardium verified the accumulation of glycosphingolipids (lower left). The scale bar is $250 \mu \mathrm{m}$. (G-H) Transmission electron microscopic examination of the myocardium revealed lamellar bodies (zebra bodies) representing lysosomes containing glycolipids, $\times 60,000$ (lower right). 
has been reported in patients with the late-onset cardiac phenotype, particular the left ventricular hypertrophy $[46,47]$. However, due to the limitations of clinical samples from cardiac biopsies or primary cardiomyocyte cultures, the investigations of the pathogenesis of FC are largely hindered. Although a variety of mutations of the $\alpha$-Gal A gene have been identified to cause Fabry disease (Human Gene Mutation Database), the pathogenetic mechanism leading to FC remains blurred. In Taiwan Fabry population with high incidence of GLA IVS4+919 G>A mutation, the underlying mechanisms that contribute to development of FC in such mutation type is also poorly understood. We therefore sought to develop a relevant human in vitro system that could be used for investigating the pathogenesis of FC and the development of its therapeutic strategies [48], especially for patients with IVS4+919 G>A mutation. In the present study, we generated the cardiomyocytes (CMs) from patient-specific iPSCs from FC patients carrying IVS4+919 G $>$ A mutation (Figure 1) to investigate the potential biomarkers for assessing the progression of Fabry-associated cardiomyocyte hypertrophy. The established patientspecific iPSC-FC-CMs not only expressed typical cardiac markers, such as TNNT2 and MYL2, but also

A
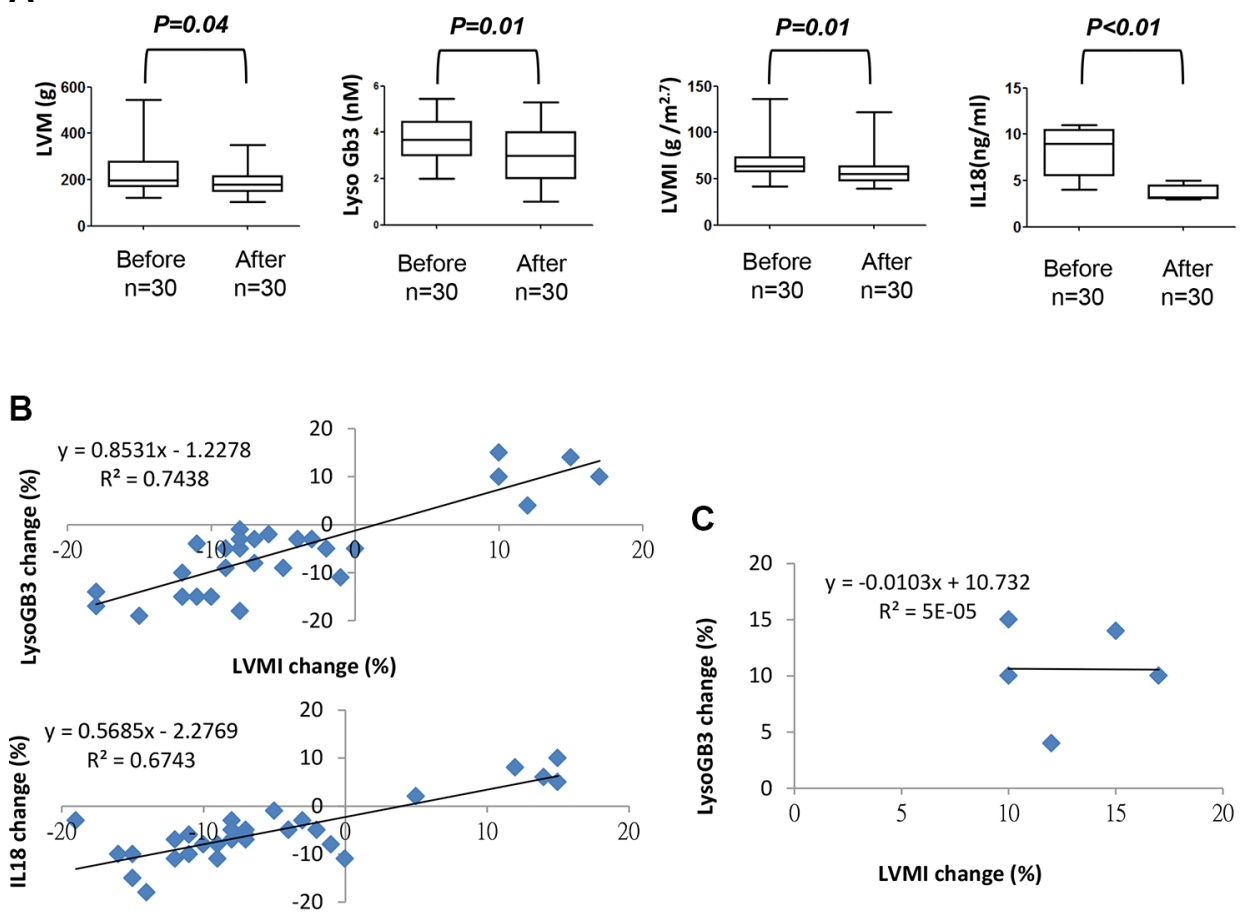

LVMI change (\%)
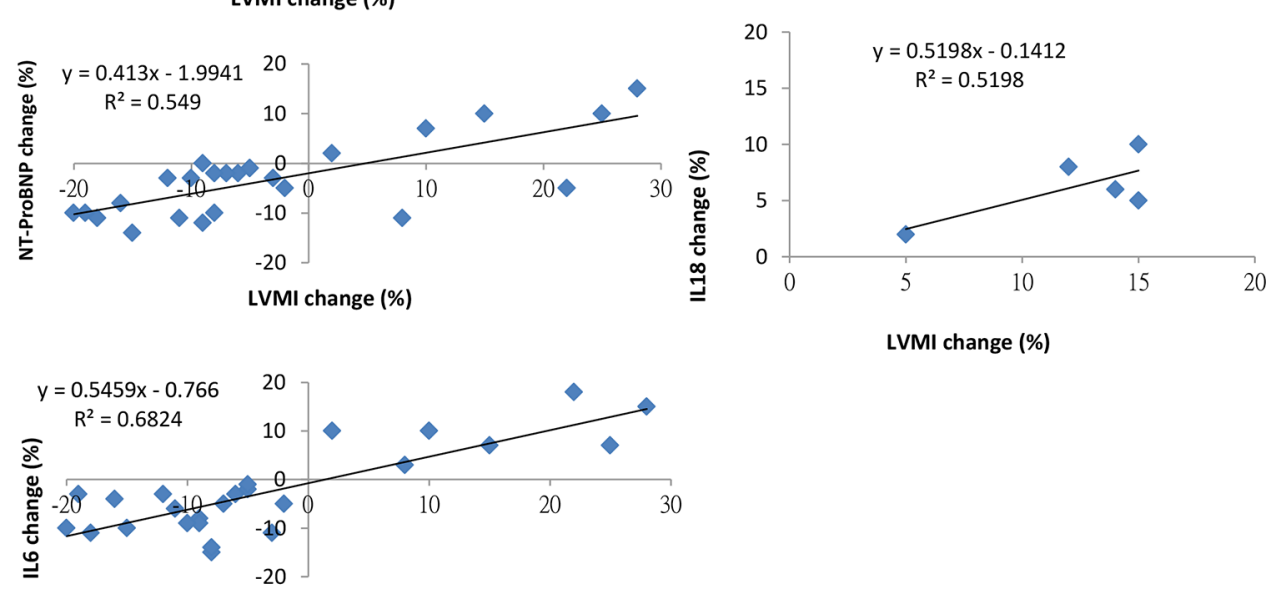

LVMI change (\%)

Figure 6: Comparison of secretory IL-18, LysoGb3 and LVH severity in FC patients before and after ERT treatment. (A) Transthoracic echocardiography parameters and lysoGb3 and IL-18 levels in 30 FC patients before and after ERT. (B) The linear regression between changes in the left ventricular mass index and changes in the levels of the biomarkers in all 30 FC patients with ERT and $(\mathbf{C})$ in 5 FC patients with LVH progression. 
recapitulated FC characters including the IVS4+919 G>A mutation and lysosomal Gb3 accumulation, decreased GLA enzyme activity and cardiomyocyte hypertrophy (Figure 2), indicating such cells could be used as a feasible experimental platform to study the cardiac functions and signaling in FC. With respective to the results of IPA (Figure 4), IL-18 has been considered to play an important role in progression of hypertrophic cardiomyopathy. Serum samples and biopsied myocardium samples from patients with late-onset $\mathrm{FC}$ further confirmed that IL-18 expression was upregulated and its secretion was in circulation at high levels, which suggests that the IL-18related pathway is activated in FC. In addition, in some Fabry patients with poor response to ERT, IL-18 but not lysoGb3 exhibited better correlation with all detected transthoracic echocardiography parameters. These findings indicated that IL-18 might represent a diagnostic marker in Fabry patients who carried IVS4+919 G>A mutation and have developed LVH.

Accumulating evidences indicate the therapeutic effects of neutralizing anti-IL-18 antibody and IL-18 binding protein (IL-18BP) in various autoimmune diseases, inflammatory bowel disease, sepsis, and acute kidney injury [49]. Because of the deteriorated role of IL-18 in cardiovascular disorders, blockage of the IL-18induced inflammatory responses has been considered the potential therapeutic strategy for vascular dysfunction and heart failure, e.g. LPS-induced myocardial dysfunction [50]. Notably, neutralization of IL-18 has been suggested to restore the endothelial progenitor cell dysfunction in systemic lupus model [51] and to inhibit neointimal formation in rats with vascular injury [52]. In addition, the ischemia/reperfusion-induced myocardial injury was ameliorated by neutralizing IL-18 with either anti-IL-18 antibody [16] or IL-18BP [53]. Moreover, neutralization of IL-18 has been shown to partially prevent hypoxiainduced cardiac hypertrophy in mice [54]. Collectively, these studies demonstrated the protective effect of IL-18 neutralizing molecules, i.e. anti-IL-18 antibody or IL-18BP, on experimental models of cardiomyopathy. In the present study, we have provided the evidences that IL-18 play a deteriorated role in Fabry-associated cardiomyopathy and LVH, and the combination of ERT and IL-18 neutralization exhibited a remarkable efficacy that reduced cardiomyocyte size and hypertrophyassociated genes in FC-iPSC-CMs. These findings
A
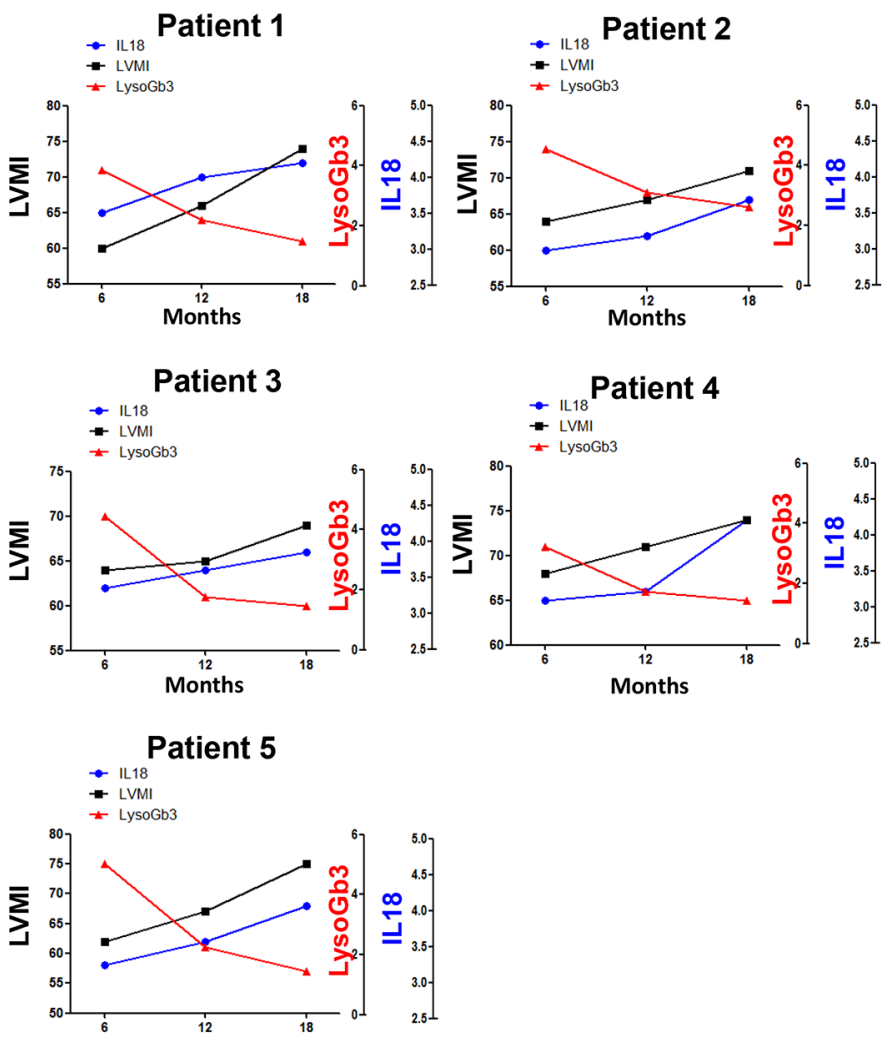

B

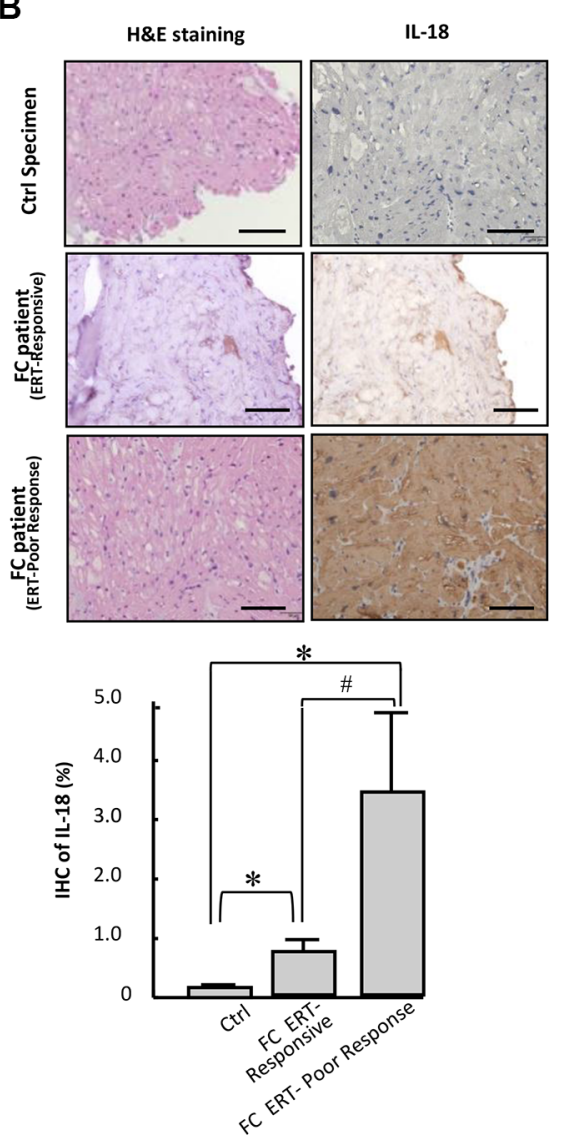

Figure 7: Serum levels and cardiac expression pattern of IL-18 in FC patients with poor response to ERT. (A) The changes of levels of IL-18 and LysoGb3 and the progression of LVH in 5 FC patients with poor response to ERT. (B) Immunohistochemistry indicated IL-18 upregulation in myocardial biopsy samples from FC patients with poor responses to ERT compared with those who responded well to ERT. 
demonstrated that IL-18 is not only a novel diagnostic biomarker but also a potential therapeutic target for the progression of FC. Neutralization of IL-18 plus conventional ERT treatment would be a beneficial synergistic approach for patients with progressive Fabry cardiomyopathy and poor response to ERT treatment, particularly in Fabry patients with IVS4+919 G>A mutation.

The limited efficacy of ERT drugs on myocardial hypertrophy have been reported by various studies. A clinical study demonstrated that long-term treatment of ERT drugs could slow down the progression of left ventricular hypertrophy. It is noticeable that no patients with LVH at treatment onset exhibited a decrease in left ventricular mass after 10 years of ERT [41]. Our previous study found that the Gb3 deposits in cardiomyocytes are cleared in patient with Fabry disease receiving ERT for more than 3 years but the hypertrophy of cardiomyocyte still presented [42]. These findings suggested that deposit of Gb3 might trigger some self-sustained mechanism(s) to induce left ventricular hypertrophy. Our present study suggested that IL-18 might play a role in one of these processes. Several preclinical studies have reported the effective clearance of microvascular endothelial accumulation of Gb3 in various organs including kidneys, heart and skin $(37,55,56)$, whereas Thurberg et al. and Keslová-Veselíková et al. consistently reported the poor histological clearance in the cardiomyocytes isolated from Fabry patients by ERT $[56,57]$. Despite these published data of ERT treatment on Gb3 deposit, the ranges of cardiac Gb3 clearance are highly variable from patient to patient. In addition, although the restorative effects of
A

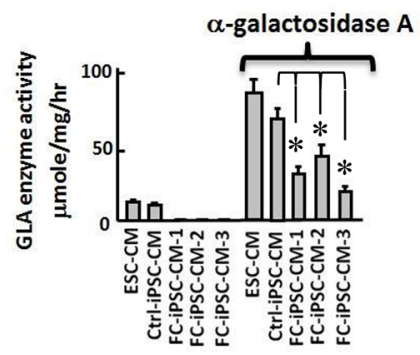

B

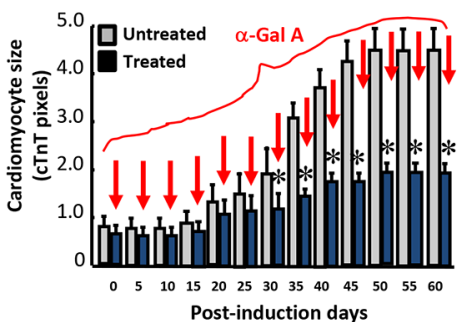

C

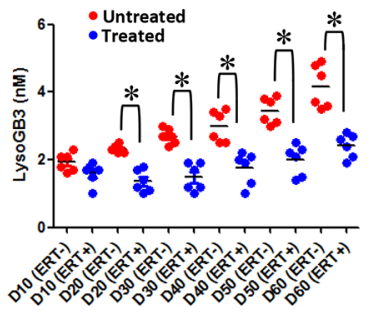

D

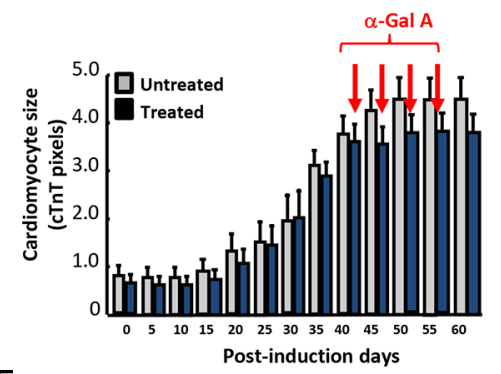

E
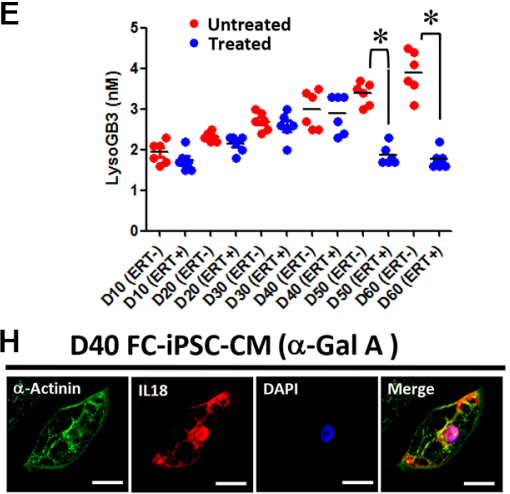

I

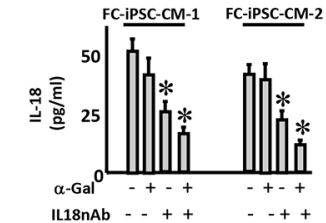

$\mathbf{F}$

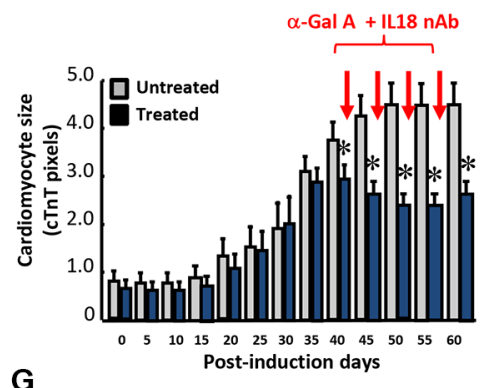

G

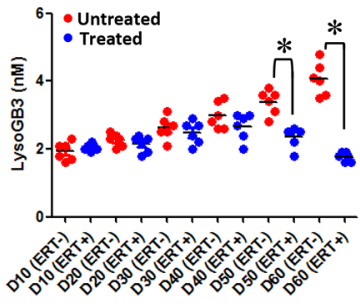

D40 FC-iPSC-CM ( $\alpha$-Gal A + IL-18 nAb)
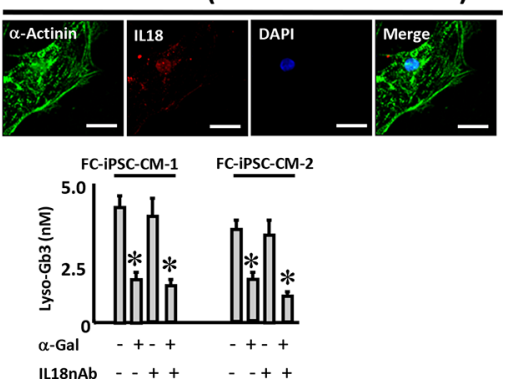

Figure 8: A combination of the IL-18 neutralization and ERT enhances the efficacy of ERT in FC-iPSC-CMs. (A) Increased GLA activity in ESC-CMs, Ctrl-iPSC-CMs and various clones of FC-iPSC-CM after the administration of alpha-galactosidase A. (B) The effect of alpha-galactosidase A administration at the onset of the cardiac induction period on cardiomyocyte size. (C) The effect of alpha-galactosidase A administration at the onset of the cardiac induction period on lysoGb3 secretion into the culture medium. (D) A protocol for the combination therapy of IL-18 neutralization and alpha-galactosidase A in FC-iPSC-CMs. (D) The effect of alphagalactosidase A administration on cardiomyocyte size at day 40 of the cardiac induction period. (E) The effect of alpha-galactosidase A administration on lysoGb3 secretion into the culture medium at day 40 of the cardiac induction period. (F) The effect of the combination of alpha-galactosidase A plus IL-18 neutralization on cardiomyocyte size at day 40 of the cardiac induction period. (G) The effect of the combination of alpha-galactosidase A plus IL-18 neutralization on lysoGb3 secretion into the culture medium at day 40 of the cardiac induction period. (H) Immunofluorescence indicating the effects of alpha-galactosidase A alone and the combination of the two agents on the expression of IL-18 in the FC-iPSC-CM platform. Scale bar $=10 \mu \mathrm{m}$. (I) ELISA indicating the effects of alpha-galactosidase A alone and the combination of the two agents on the production of IL-18 and LysoGb3 from the FC-iPSC-CM platform. 
ERT on cardiac functions reported in these preclinical data are likely to be secondary to ERT efficacy on vascular endothelial cells, some controversial data have also been reported. In mouse model of Fabry disease, both non-phosphorylated alpha-galactosidase A produced from moss and phosphorylated alpha-galactosidase A were capable of effective clearance of Gb3 in various tissues, including heart, kidney and liver [34] Alternative explanations for the poor Gb3 clearance in heart tissue have also been proposed. One possible explanation could be the difficulty in the detection of Gb3 reduction at the microscopic levels in cardiomyocytes from the endomyocardial biopsy samples. Another possibility could be that, the clearance of Gb3 in cardiomyocytes will require longer periods of ERT treatment, since that Gb3 deposit in terminally differentiated cardiomyocytes stands for the lifetime of substrate accumulation, [56]. Recent evidence showed in vitro drug efficacy that directly cleared lysosomal Gb3 with substrate reduction therapy (SRT) drug via glucosylceramide synthase inhibition [31], in which the drug efficacy did not involve any reduction of the Gb3 deposit in microvascular endothelium. In the present study, our findings have indicated that, administration of Replagal $(5 \mu \mathrm{g} / \mathrm{ml})$ at the onset of cardiac differentiation for 60 days substantially prevented

A

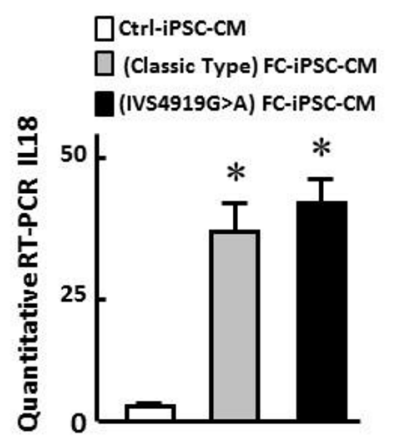

B

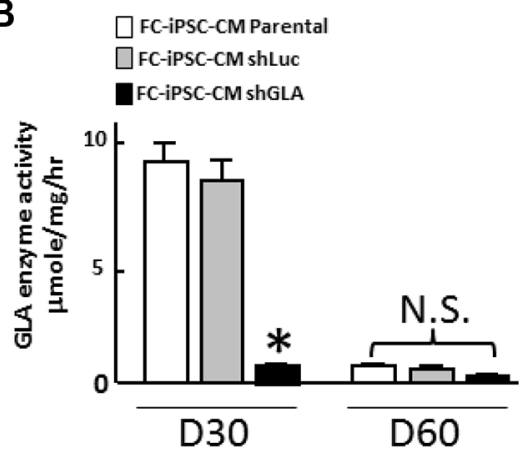

the development of cardiomyocyte hypertrophy during the differentiation course (Figure 8B). However, the same treatment failed to restore the cardiomyocyte hypertrophy at post-induction 40 days, a stage of FC-iPSC-CMs that have exhibited severe phenotype of FC, (Figure 8D). Our data supported that early treatment of ERT drug may possess high potential to ameliorate or prevent Fabry cardiomyopathy and other cardiac co-morbidities in Fabry patients.

The mechanisms underlying the upregulation and elevated secretion of IL-18 in FC-iPSC-CMs were assessed, and one of possible cause was the reduced expression and activity of GLA in Fabry disease. In our GLA knockdown study, we only observed a moderate increase in IL-18 secretion by GLA knockdown at postinduction 30 days, but this secretion was indistinguishable among all groups with any given treatment at postinduction 60 days, a stage fully matured FC-iPSCCMs that exhibited all FC-specific characteristics. These data suggested that low GLA expression might not be the predominant factor that stimulates the IL-18 secretion. Rather than low GLA content and Gb3 deposit, other factors generated during the long course of Fabry cardiomyopathy may also contribute to the high IL-18 secretion. Chévrier et al. have reported that
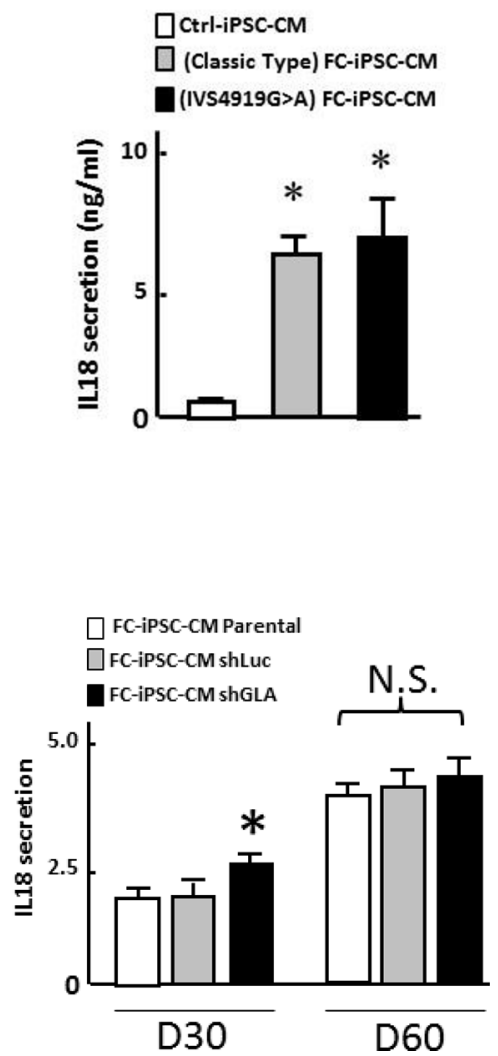

Figure 9: Evaluation of the interrelationship of GLA activity and IL-18 secretion. (A) Comparison of GLA activity and IL-18 secretion patterns in FC-iPSC-CMs derived from Fabry cohorts with GLA IVS4+919 G>A mutation or classic type mutation. (B) Effect of GLA knockdown using shRNA against GLA on GLA activity and IL-18 at post-induction 30 days and 60 days. Data shown here is the mean $\pm \mathrm{SD}$ of three independent experiments, ${ }^{*} P<0.05$ vs. parental and ShLuc. These experiments were conducted in FC-iPSC$\mathrm{CM}$ derived from two individual FC patients. 
normal autophagic flux is impaired in Fabry disease [58]. Our previous work also demonstrated that several pro-inflammatory cytokines were also involved in the development of Fabry cardiomyopathy carrying IVS4+919 $\mathrm{G}>\mathrm{A}$ mutation [38]. The mechanisms that links to the high IL-18 secretion in FC-iPSC-CMs remain not fully understood, and future works will be required to elucidate the mechanisms responsible for the regulation of IL-18 secretion in Fabry cardiomyopathy with IVS4+919 G>A mutation.

In the present study, among the 25 recruited Fabry patients who had developed $\mathrm{FC}$ for data acquisition and analysis as well as iPSC studies, only 1 female patient with FC was recruited $(1 / 25$; women no. $=4 \%)$. The major limitation of this study could be that, the patients were also not randomly selected for participation and may not represent the entire population of Fabry patients carrying GLA IVS4+919 G>A mutation. Actually, asymptomatic women were unlikely to be recruited and assigned for the treatment of Fabry disease and its co-morbidities. Previously, Kampmann et al. have reported a large crosssectional and long longitudinal study of cardiomyopathy in Anderson-Fabry disease [59]. Although there was a strong correlation exists between age and LVM in both hemi and heterozygotes, the onset for the increase of LVM in males was approximately 10 years earlier than in that in females and the increase rate in male is also greater than that in females. Presumably, the slower and variable progression rate of LVM in females has been attributed to the different distribution of Gb3 in cardiomyocytes comparing to males. As for the mutation type reported in our present study, the causes leading to low recruitment rate of female Fabry patients with cardiomyopathy in Fabry patients with IVS4+919 G>A mutation is uncertain. Further approaches and clinical studies will be required to elucidate whether IL-18 also serves a critical role in the pathogenesis of Fabry cardiomyopathy in female patients.

In conclusion, FC-iPSC-CMs represent a novel in vitro model that recapitulates the cardiac phenotype of Fabry patients. Combined with proteomic analysis, FCiPSC-CMs offer a promising platform for investigating the potential biomarkers of FC. Moreover, using biopsy and serum samples, we have confirmed our findings using FCiPSC-CMs from our clinical patients. In addition, in our in vitro iPSC-CM model, the combination of ERT plus IL-18 neutralization effectively ameliorated and prevented the progression of hypertrophy and cardiomyopathy in hypertrophic FC-iPSC-CMs (Figure 8). Finally, our data have illustrated the important role of IL-18 in Fabryassociated cardiac manifestations in Fabry patient carrying GLA IVS4+919 G>A mutation and identified the IL-18 pathway as a potential therapeutic option that should be further elucidated in animal studies and clinical cases of patients.

\section{MATERIALS AND METHODS}

\section{Human iPSC generation and cultivation}

To generate integration-free iPSCs, cells were nucleofected with $3 \mu \mathrm{g}$ expression plasmid mixture using Amaxa $^{\mathrm{TM}}$ human $\mathrm{T}$ Cell Nucleofector ${ }^{\mathrm{TM}} \mathrm{Kit}$ (Lonza). In each nucleofection, $0.83 \mu \mathrm{g}$ PCXLE-hOCT3/4shp53, $0.83 \mu \mathrm{g}$ PCXLE-hSK, $0.83 \mu \mathrm{g}$ pCXLE-hUL, and $0.5 \mu \mathrm{g}$ pCXWB-EBNA1 were used. $3 \times 10^{6}$ cells were nucleofected with Amaxa Nucleofector II using program V-024. Cells were cultured the exactly same way as for reprogramming with lentiviral vector expect that every 10-14 days, freshly thawed inactivated mouse embryonic fibroblasts (MEFs) feeder cells were added into each dish. Undifferentiated iPSCs were maintained on inactivated MEFs $\left(50,000\right.$ cells $\left./ \mathrm{cm}^{2}\right)$ in human ESC medium (DMEM/ F12 (Gibco) supplemented with 20\% KnockOut serum replacer (KSR; Invitrogen), $0.1 \mathrm{mM}$ non-essential amino acids (Invitrogen), $1 \mathrm{mM}$ L-glutamine, $0.1 \mathrm{mM}$ ß-mercaptoethanol, $10 \mathrm{ng} / \mathrm{ml}$ recombinant human basic fibroblast growth factor (bFGF), and antibiotics (Gibco).

\section{iPSC-derived cardiomyocytes}

Cells were dissociated by Versene (Life technologies), then incubate the plate at $37^{\circ} \mathrm{C}, 5 \% \mathrm{CO} 2$ and wait for 4 minutes. Aspirate the supernatant, resuspend the cells in mTeSR $1+5 \mu \mathrm{M}$ Y27632 and seeded onto Geltrex-coated plates at a density of $3 \times 105$ cells $/ \mathrm{cm} 2$. Add mTeSR $1+5 \mu \mathrm{M}$ Y27632 medium to each well to make a final volume of $2 \mathrm{ml}$ in each well of the 12-well plate. This time point corresponds to day -4 . On day -3 , day -2 , and day -1 , aspirate the medium and replace with $2 \mathrm{ml}$ room temperature $\mathrm{mTeSR} 1$ per well of the 12well plate. At on day 0 , the cells were treated with $6 \mu \mathrm{M}$ CHIR99021 (Selleckchem) in insulin-free RPMI/B27 without-insulin medium (Life Technologies) for 24 hours. The medium was replaced with basal medium for another 2 days. At on day 3, the culture medium was subsequently replaced with $5 \mu \mathrm{M}$ IWP2 (Tocris) in insulin-free RPMI/ B27 without-insulin for 48 hours. At On day 7, the culture medium was changed to RPMI/B27 with containing insulin (Life Technologies), and the culture medium was refreshed thereafter every 3 days.

\section{Alkaline phosphatase staining}

First, removed the culture medium and washed by $1 \times$ PBS twice, then cells were fixed with $80 \%$ alcohol for at least 2 hours at $4^{\circ} \mathrm{C}$. To aspirate the alcohol and infiltrated by double-distilled water and then for 2 to 3 minutes. Remove the double-distilled water and add $100 \mathrm{mM}$ Tris$\mathrm{HCl}(\mathrm{pH} \mathrm{8.2-8.5)} \mathrm{for} 5 \mathrm{~min}$. Remove Tris- $\mathrm{HCl}$ and add 
alkaline phosphatase substrate working solution (Vector) for 1 hour. Colonies stained purple indicated positive alkaline phosphatase activity.

\section{Reverse transcription-polymerase chain reaction (RT-PCR)}

Total RNA was isolated with TRIzol Reagent (Invitrogen) and quantified by spectrophotometry at 260 nm. On a GeneAmpÒ PCR System 9700 thermocycler (Applied Biosystems), $3 \mu \mathrm{g}$ of each total RNA was reverse- transcribed with SuperScript III (Invitrogen) at $55^{\circ} \mathrm{C}$ for $1 \mathrm{~h}$ into total complementary DNA, which was then used as the template for the subsequent PCR reactions and analysis. The PCR reactions involved an initial denaturation at $94^{\circ} \mathrm{C}$ for $5 \mathrm{~min}$, followed by 25 or 30 cycles at $94^{\circ} \mathrm{C}$ for $30 \mathrm{~s}$, exposure to an appropriate annealing temperature $\left(58-62^{\circ} \mathrm{C}\right)$ for $30 \mathrm{~s}$, and then a final incubation at $72^{\circ} \mathrm{C}$ for $45 \mathrm{~s}$. The primers and cycling conditions for real-time RT-PCR were shown in Table 1. Amplified RT- PCR products were then analyzed on $2 \%$ agarose gels and visualized using ethidium bromide staining and a camera system (Transilluminator/SPOT; Diagnostic Instruments). The gel images of the RTPCR products were directly scanned (ONEDscan 1-D Gel Analysis Software; Scanalytic Inc.), and the relative densities were obtained by determining the ratio of the signal intensity to the GAPDH or $\beta$-actin.

\section{Genomic DNA extraction and sequence for identifying the genetic mutation}

The genomic DNA extraction from iPSC-WT, iPSCFabry, CM-WT and CM-Fabry was follow the manuscript of QIAamp DNA Mini Kit. DNA concentrations were determined using a Nanodrop spectrophotometer (Infinigen, USA). The IVS4+919 G>A mutations was characterized by genomic DNA sequencing. The genomic DNA from various cell lines was performed two different stages of PCR to amplify the mutation site for further sequencing.

\section{Immunofluorescence staining}

Aspirate the medium and wash cells twice with PBS. First, cells were fixed with $1 \%$ (vol/vol) paraformaldehyde for 10 minutes then aspirate the paraformaldehyde. Second, fixed with $70 \%$ alcohol for 10 minutes at room temperature then aspirate the alcohol. Add $0.1 \%$ NP-40 (Sigma \#18896-50 ml) for 20 minutes then wash twice with PBS. To block cells with blocking solution (PBS with $0.3 \% \mathrm{BSA}$ and $5 \%$ serum) for 30 minutes to 1 hour. To aspirate the blocking solution then stain cell with primary antibodies in the blocking solution overnight at $4 \mathrm{C}$. Cells washed three times in PBS, then stained with secondary antibodies at 1:200 in PBS for $1 \mathrm{~h}$ at room temperature.
Cells were washed three times in PBS, and nuclei were stained with Hoechst 33342 (Life Technologies) at 1:5000 in PBS for $5 \mathrm{~min}$ at room temperature. Prior to imaging, cells were inverted onto a glass coverslip containing one drop of SlowFade Gold antifade reagent (millipore) and sandwiched with another glass coverslip on top. DAPI was used as nuclear stain (blue). Images were obtained using fluorescent microscopy and a digital camera.

\section{Western blotting}

Cells were lysed in RIPA lysis buffer ( $0.5 \mathrm{M}$ Tris$\mathrm{HCl}, \mathrm{pH} 7.4,1.5 \mathrm{M} \mathrm{NaCl}, 2.5 \%$ deoxycholic acid, $10 \%$ NP-40, $10 \mathrm{mM}$ EDTA, protease inhibitor), and the protein lysates were subjected to SDS-PAGE followed by electronblotting onto a PVDF membrane. Membranes were probed with monoclonal antibodies against $\alpha$-galactosidase A (GeneTex) and $\beta$-actin (sigma). Bands were visualized by chemilum inescence detection reagents.

\section{GLA enzyme activity}

Cells were washed twice with PBS. Afterward, cells were lysed in $60 \mu \mathrm{l}$ lysis buffer (N-acetyl-D-galactosamine, 4-methylumbelliferyl- $\alpha$-D-galactopyranoside (4-MUGal), $0.5 \%$ Triton X-100). A 4-methylumbelliferone (4-MU) standard curve ranging from $0.15 \mu \mathrm{M}$ to $5000 \mu \mathrm{M} .10 \mu \mathrm{l}$ lysate were added to $50 \mu \mathrm{l}$ Assay Buffer, containing $6 \mathrm{mM}$ 4-MUGal and $117 \mathrm{mM} \mathrm{N}$-acetyl-D-galactosamine, and incubated at $37^{\circ} \mathrm{C}$ for $1 \mathrm{hr}$. Next, $70 \mu \mathrm{l}$ glycine-carbonate solution was then added to stop the reaction. At last, using a fluorescence with excitation and emission wavelength of $365 \mathrm{~nm}$ and $448 \mathrm{~nm}$.

\section{Karyotyping}

The chromosome number and structure were analyzed following the previous description [33]. Briefly, the iPSCs were treated with Colcemid (Gibco) to arrest cells in metaphase, and then harvested within the hypotonic solution to spread the chromosomes. Following fixation with methanol and acetic acid mixture (3:1), the cells were squashed on the slide and then stained with the Wright staining solution (Sigma-Aldrich). Afterwards, the chromosome G-banding were visualized, photographed, and arranged into the karyogram at the TVGH cytogenetics laboratory.

\section{Transmission electron microscopy (TEM)}

Cells were suspended in $1.2 \%$ agarose in $0.1 \mathrm{M}$ phosphate buffer $(\mathrm{pH} 7.4)$, and fixed with $2 \%$ paraformaldehyde and $2.5 \%$ glutaraldehyde at $4{ }^{\circ} \mathrm{C}$ overnight. Following washing with the phosphate buffer, the samples were post-fixed with $1 \% \mathrm{OsO}_{4}$ in $0.05 \mathrm{M}$ phosphate buffer at RT for $1 \mathrm{hr}$. After washing with 
distilled water, the samples were "en bloc" staining with $0.2 \%$ uranyl acetate in $70 \% \mathrm{EtOH}$ at $4^{\circ} \mathrm{C}$ overnight. The samples were dehydrated in a serial dilution of ethanol for $10 \mathrm{~min}$ each (from $70 \%$ to $100 \%$ ethanol) and further infiltrated with a $100 \%$ ethanol/acetone (1:1) mixture and $100 \%$ acetone for $15 \mathrm{~min}$ each. Then, the samples were embedded by Spurr's epoxy resin. Briefly, the samples were infiltrated with $100 \%$ acetone/epoxy resin $(1: 1)$ and (1:3) mixture for $1 \mathrm{hr}$ each, and then transfer to epoxy resin for continuous infiltration for $24 \mathrm{hr}$. The epoxy resin was polymerized and solidified at $72^{\circ} \mathrm{C}$ for $72 \mathrm{hr}$. Following the polymerization, the resin blocks were trimmed and sliced to $80 \mathrm{~nm}$ sections using an ultramicrotome (Leica EM UC7, Vienna, Austria). Thin sections were transferred to 200 mesh copper grids and counterstained with $2.5 \%$ uranyl acetate for $30 \mathrm{~min}$ and $0.4 \%$ lead citrate for 4 min prior to observation with a JEM-2000EXII electron microscope (JEOL USA, Inc., Massachusetts, USA).

\section{Microarray analysis}

Total RNA was extracted from 40 days postdifferentiated cardiomyocytes by using TRIzol Reagent (Invitrogen) and then sent to the Microarray core laboratory of TVGH for the following cDNA synthesis, labeling, hybridization, and scanning. The GeneChip ${ }^{\circledR}$ Human Gene array from Affymetrix was used and the data normalization, compare, and analysis were conducted by Affymetrix Power Tools. The differentially expressed genes ( $\geq 2$-folds changes) between control and Fabry sample were isolated. Heat map was visualized by MultiExperiment Viewer (MeV) downloaded from the TM4 microarray software suite (http://www.tm4.org/). The color present the normalized expression value of $\log 2$ (fold change), and the red indicates up-regulated genes and the blue indicates the down-regulated genes.

\section{Toluidine blue staining}

Tissue specimens of patients with FC were collected and retrieved from the archives of the Department of Pathology of Taipei Veterans General Hospital. The tissue sections were performed Toluidine blue staining after deparaffinization, rehydration, and wash. Briefly, $0.1 \%$ Toluidine blue was used to stain the tissue sections. Following the staining, the sections were washed in distilled water three times, dehydrated quickly through 95\% and absolute $\mathrm{EtOH}$, cleared in Xylene, and mounted for analysis.

\section{Quantitative RT-PCR (qPCR)}

Following total RNA isolation and cDNA synthesis, the qPCR analysis using Power SYBR-Green PCR master mix (Applied Biosystems) was performed in LightCycler 480 instrument (Roche Diagnostics $\mathrm{GmbH}$ ). Melting curve analysis was used to confirm the amplification specificity. $\beta$-actin was used as an internal control. The relative gene expression levels were determined in comparison with the samples of Ctrl-iPSC-CM.

\section{Immunohistochemistry (IHC)}

The formalin-fixed and paraffin-embedded tissues were sectioned to $4-\mu$ m-thick for IHC analysis of the IL18 expression. Briefly, the tissue sections were deparaffinized with xylene, rehydrated through a series of graded ethanol solutions $(100 \%, 90 \%, 75 \%)$, washed with PBS, and retrieved the antigen with $10 \mathrm{mM}$ citrate buffer $(\mathrm{pH} 6.0)$ for $40 \mathrm{~min}$ under boiling. The Envision detection system (Dako Cytomation, Glostrup, Denmark) was purchase for IHC staining. Following to the manufacture's instruction, the sections were treated with peroxidase-blocking solution, blocked non-specific binding with $10 \%$ nonimmune goat serum, probed with the IL18 primary antibody (abcam; ab68435) at RT overnight, stained with peroxidase/DAB + , counterstained with Mayer's hematoxylin, and then mounted for analysis.

\section{Protocol for enzyme replacement therapy (ERT) in vitro and preclinical studies}

For the in vitro ERT treatment, the cardiomyocytes were treated with $5 \mu \mathrm{g} / \mathrm{ml}$ Replagal. The in vitro concentrations of Replagal is the theoretical maximal plasma concentrations of agalsidase alpha in infused Fabry patients receiving approved doses [34]. The culture medium containing with Replagal were refreshed every two days. Four days post-treatment, the cells were collected for the following analysis. In addition, the enzyme replacement therapy (ERT) administration in patients with FC was supported by Ministry of Health and Welfare in Taiwan based on Gb3 accumulation in cardiac biopsy, and executed by intravenously administration of Agalsidase alfa (Replagal ${ }^{\circledR} 0.2 \mathrm{mg} / \mathrm{kg}$ ) or agalsidase beta (Fabrazyme ${ }^{\circledR} 1.0 \mathrm{mg} / \mathrm{kg}$ ) at a two-week interval for an average of 15.5 months (median; range 6-24). Echocardiography and CMR were used to validate the ERT efficacy and prognosis.

\section{Statistical analysis}

For the human subject data, the variables are presented as the mean \pm standard deviation and compared with Student's $t$-test. A paired $t$-test was used to evaluate ERT efficacy. We used a linear regression model to explore the associations between the changes in the levels of the biomarkers and left ventricular mass index (LVMI) before and after ERT. The statistical analyses were performed with SPSS software version 13.0 (SPSS, Inc., Chicago, IL, USA). The results were considered to be significant at $P<0.05$. A more detailed description of the methods used in this study can be found in the Online Appendix. 


\section{ACKNOWLEDGMENT AND FUNDING}

This research was supported by Academia Sinica and Ministry of Science and Technology, MOST (1040210-01-09-02, 105-0210-01-13-01, 105-2633-B-009-003, 105-2325-B-010-005, and 105-3011-B010-001), Ministry of Health and Welfare (MOHW105-TDU-B-211-133017, MOHW105-TDU-B-211-134003), Research Foundation of Cardiovascular Medicine, and Taipei Veterans General Hospital (V103C-166, V104C-175, V104E14-001-MY3-2, V105C-077, V105C-189).

\section{CONFLICTS OF INTEREST}

None.

\section{REFERENCES}

1. Germain DP. Fabry disease. Orphanet J Rare Dis. 2010; $5: 30$.

2. Weidemann F, Niemann M, Breunig F, Herrmann S, Beer M, Stork S, Voelker W, Ertl G, Wanner C, Strotmann J. Long-term effects of enzyme replacement therapy on fabry cardiomyopathy: evidence for a better outcome with early treatment. Circulation. 2009; 119:524-529.

3. Togawa T, Kawashima I, Kodama T, Tsukimura T, Suzuki T, Fukushige T, Kanekura T, Sakuraba H. Tissue and plasma globotriaosylsphingosine could be a biomarker for assessing enzyme replacement therapy for Fabry disease. Biochem Biophys Res Commun. 2010; 399:716-720.

4. Whitfield PD, Calvin J, Hogg S, O'Driscoll E, Halsall D, Burling K, Maguire G, Wright N, Cox TM, Meikle PJ, Deegan PB. Monitoring enzyme replacement therapy in Fabry disease - role of urine globotriaosylceramide. J Inherit Metab Dis. 2005; 28:21-33.

5. Vedder AC, Linthorst GE, van Breemen MJ, Groener JE, Bemelman FJ, Strijland A, Mannens MM, Aerts JM, Hollak CE. The Dutch Fabry cohort: diversity of clinical manifestations and Gb3 levels. J Inherit Metab Dis. 2007; 30:68-78.

6. Liu HC, Lin HY, Yang CF, Liao HC, Hsu TR, Lo CW, Chang FP, Huang CK, Lu YH, Lin SP, Yu WC, Niu DM. Globotriaosylsphingosine (lyso-Gb3) might not be a reliable marker for monitoring the long-term therapeutic outcomes of enzyme replacement therapy for late-onset Fabry patients with the Chinese hotspot mutation (IVS4+919G $>$ A). Orphanet J Rare Dis. 2014; 9:111.

7. O’Brien LC, Mezzaroma E, Van Tassell BW, Marchetti C, Carbone S, Abbate A, Toldo S. Interleukin-18 as a therapeutic target in acute myocardial infarction and heart failure. Mol Med. 2014; 20:221-229.

8. Arend WP, Palmer G, Gabay C. IL-1, IL-18, and IL-33 families of cytokines. Immunol Rev. 2008; 223:20-38.
9. Born TL, Thomassen E, Bird TA, Sims JE. Cloning of a novel receptor subunit, AcPL, required for interleukin-18 signaling. J Biol Chem. 1998; 273:29445-29450.

10. Torigoe K, Ushio S, Okura T, Kobayashi S, Taniai M, Kunikata T, Murakami T, Sanou O, Kojima H, Fujii M, Ohta T, Ikeda M, Ikegami $H$, et al. Purification and characterization of the human interleukin-18 receptor. J Biol Chem. 1997; 272:25737-25742.

11. Okamura H, Tsutsi H, Komatsu T, Yutsudo M, Hakura A, Tanimoto T, Torigoe K, Okura T, Nukada Y, Hattori K, et al. Cloning of a new cytokine that induces IFN-gamma production by T cells. Nature. 1995; 378:88-91.

12. Dinarello CA, Novick D, Puren AJ, Fantuzzi G, Shapiro L, Muhl H, Yoon DY, Reznikov LL, Kim SH, Rubinstein M. Overview of interleukin-18: more than an interferon-gamma inducing factor. J Leukoc Biol. 1998; 63:658-664.

13. Wang M, Markel TA, Meldrum DR. Interleukin 18 in the heart. Shock 2008; 30:3-10.

14. Platis A, Yu Q, Moore D, Khojeini E, Tsau P, Larson D. The effect of daily administration of IL-18 on cardiac structure and function. Perfusion. 2008; 23:237-242.

15. Yu Q, Vazquez R, Khojeini EV, Patel C, Venkataramani R, Larson DF. IL-18 induction of osteopontin mediates cardiac fibrosis and diastolic dysfunction in mice. Am J Physiol Heart Circ Physiol 2009; 297:H76-85.

16. Venkatachalam K, Prabhu SD, Reddy VS, Boylston WH, Valente AJ, Chandrasekar B. Neutralization of interleukin-18 ameliorates ischemia/reperfusion-induced myocardial injury. J Biol Chem. 2009; 284:7853-7865.

17. Mallat Z, Henry P, Fressonnet R, Alouani S, Scoazec A, Beaufils P, Chvatchko Y, Tedgui A. Increased plasma concentrations of interleukin-18 in acute coronary syndromes. Heart. 2002; 88:467-469.

18. Naito Y, Tsujino T, Fujioka Y, Ohyanagi M, Okamura H, Iwasaki T. Increased circulating interleukin-18 in patients with congestive heart failure. Heart. 2002; 88:296-297.

19. Mallat Z, Heymes C, Corbaz A, Logeart D, Alouani S, Cohen-Solal A, Seidler T, Hasenfuss G, Chvatchko Y, Shah AM, Tedgui A. Evidence for altered interleukin 18 (IL)-18 pathway in human heart failure. FASEB J. 2004; 18:1752-1754.

20. Chien Y, Liao YW, Liu DM, Lin HL, Chen SJ, Chen HL, Peng CH, Liang CM, Mou CY, Chiou SH. Corneal repair by human corneal keratocyte-reprogrammed iPSCs and amphiphatic carboxymethyl-hexanoyl chitosan hydrogel. Biomaterials. 2012; 33:8003-8016.

21. Chou SJ, Tseng WL, Chen CT, Lai YF, Chien CS, Chang YL, Lee HC, Wei YH, Chiou SH. Impaired ROS Scavenging System in Human Induced Pluripotent Stem Cells Generated from Patients with MERRF Syndrome. Sci Rep. 2016; 6:23661.

22. Chang YC, Chang WC, Hung KH, Yang DM, Cheng YH, Liao YW, Woung LC, Tsai CY, Hsu CC, Lin TC, Liu JH, Chiou $\mathrm{SH}$, Peng $\mathrm{CH}$, et al. The generation of induced 
pluripotent stem cells for macular degeneration as a drug screening platform: identification of curcumin as a protective agent for retinal pigment epithelial cells against oxidative stress. Front Aging Neurosci. 2014; 6:191.

23. Robinton DA, Daley GQ. The promise of induced pluripotent stem cells in research and therapy. Nature. 2012; 481:295-305.

24. Drawnel FM, Boccardo S, Prummer M, Delobel F, Graff A, Weber M, Gerard R, Badi L, Kam-Thong T, Bu L, Jiang X, Hoflack JC, Kiialainen A, et al. Disease modeling and phenotypic drug screening for diabetic cardiomyopathy using human induced pluripotent stem cells. Cell Rep. 2014; 9:810-821.

25. Yang T, Rubart M, Soonpaa MH, Didie M, Christalla P, Zimmermann WH, Field LJ. Cardiac engraftment of genetically-selected parthenogenetic stem cell-derived cardiomyocytes. PLoS One 2015; 10:e0131511.

26. Liang P, Lan F, Lee AS, Gong T, Sanchez-Freire V, Wang Y, Diecke S, Sallam K, Knowles JW, Wang PJ, Nguyen PK, Bers DM, Robbins RC, et al. Drug screening using a library of human induced pluripotent stem cellderived cardiomyocytes reveals disease-specific patterns of cardiotoxicity. Circulation. 2013; 127:1677-1691.

27. Del Alamo JC, Lemons D, Serrano R, Savchenko A, Cerignoli F, Bodmer R, Mercola M. High throughput physiological screening of iPSC-derived cardiomyocytes for drug development. Biochim Biophys Acta. 2016.

28. Han L, Li Y, Tchao J, Kaplan AD, Lin B, Mich-Basso J, Lis A, Hassan N, London B, Bett GC, Tobita K, Rasmusson RL, Yang L. Study familial hypertrophic cardiomyopathy using patient-specific induced pluripotent stem cells. Cardiovasc Res. 2014; 104:258-269.

29. Karakikes I, Termglinchan V, Wu JC. Human-induced pluripotent stem cell models of inherited cardiomyopathies. Curr Opin Cardiol. 2014; 29:214-219.

30. Sato Y, Kobayashi H, Higuchi T, Shimada Y, Era T, Kimura S, Eto Y, Ida H, Ohashi T. Disease modeling and lentiviral gene transfer in patient-specific induced pluripotent stem cells from late-onset Pompe disease patient. Mol Ther Methods Clin Dev. 2015; 2:15023.

31. Itier JM, Ret G, Viale S, Sweet L, Bangari D, Caron A, Le-Gall F, Benichou B, Leonard J, Deleuze JF, Orsini C. Effective clearance of GL-3 in a human iPSC-derived cardiomyocyte model of Fabry disease. J Inherit Metab Dis. 2014; 37:1013-1022.

32. Aggarwal P, Turner A, Matter A, Kattman SJ, Stoddard A, Lorier R, Swanson BJ, Arnett DK, Broeckel U. RNA expression profiling of human iPSC-derived cardiomyocytes in a cardiac hypertrophy model. PLoS One. 2014; 9:e108051.

33. Moralli D, Yusuf M, Mandegar MA, Khoja S, Monaco ZL, Volpi EV. An improved technique for chromosomal analysis of human ES and iPS cells. Stem cell reviews 2011; 7:471-477.

34. Shen JS, Busch A, Day TS, Meng XL, Yu CI, DabrowskaSchlepp P, Fode B, Niederkruger H, Forni S, Chen S,
Schiffmann R, Frischmuth T, Schaaf A. Mannose receptormediated delivery of moss-made alpha-galactosidase A efficiently corrects enzyme deficiency in Fabry mice. J Inherit Metab Dis. 2016; 39:293-303.

35. Bernstein HS, Bishop DF, Astrin KH, Kornreich R, Eng CM, Sakuraba H, Desnick RJ. Fabry disease: six gene rearrangements and an exonic point mutation in the alphagalactosidase gene. J Clin Invest. 1989; 83:1390-1399.

36. Eng CM, Niehaus DJ, Enriquez AL, Burgert TS, Ludman MD, Desnick RJ. Fabry disease: twenty-three mutations including sense and antisense $\mathrm{CpG}$ alterations and identification of a deletional hot-spot in the alphagalactosidase A gene. Hum Mol Genet. 1994; 3:1795-1799.

37. Eng CM, Guffon N, Wilcox WR, Germain DP, Lee P, Waldek S, Caplan L, Linthorst GE, Desnick RJ. Safety and efficacy of recombinant human alpha-galactosidase A -replacement therapy in Fabry's disease. N Engl J Med. 2001; 345:9-16.

38. Chen KH, Chien Y, Wang KL, Leu HB, Hsiao CY, Lai YH, Wang CY, Chang YL, Lin SJ, Niu DM, Chiou SH, Yu WC. Evaluation of Proinflammatory Prognostic Biomarkers for Fabry Cardiomyopathy With Enzyme Replacement Therapy. Can J Cardiol. 2016; 32:1221 e1221-1221 e1229.

39. Brito D, Matias JS, Sargento L, Cabral MJ, Madeira HC. Plasma N-terminal pro-brain natriuretic peptide: a marker of left ventricular hypertrophy in hypertrophic cardiomyopathy. Rev Port Cardiol. 2004; 23:1557-1582.

40. Navarrete EG, Liang P, Lan F, Sanchez-Freire V, Simmons C, Gong T, Sharma A, Burridge PW, Patlolla B, Lee AS, Wu H, Beygui RE, Wu SM, et al. Screening druginduced arrhythmia [corrected] using human induced pluripotent stem cell-derived cardiomyocytes and lowimpedance microelectrode arrays. Circulation. 2013; 128:S3-13.

41. Kampmann C, Perrin A, Beck M. Effectiveness of agalsidase alfa enzyme replacement in Fabry disease: cardiac outcomes after 10 years' treatment. Orphanet J Rare Dis. 2015; 10:125.

42. Hsu TR, Sung SH, Chang FP, Yang CF, Liu HC, Lin HY, Huang CK, Gao HJ, Huang YH, Liao HC, Lee PC, Yang AH, Chiang CC, et al. Endomyocardial biopsies in patients with left ventricular hypertrophy and a common Chinese later-onset Fabry mutation (IVS4 + 919G > A). Orphanet J Rare Dis. 2014; 9:96.

43. Desnick RJ, Wasserstein MP. Fabry disease: clinical features and recent advances in enzyme replacement therapy. Adv Nephrol Necker Hosp. 2001; 31:317-339.

44. Aerts JM, Groener JE, Kuiper S, Donker-Koopman WE, Strijland A, Ottenhoff R, van Roomen C, Mirzaian M, Wijburg FA, Linthorst GE, Vedder AC, Rombach SM, CoxBrinkman J, et al. Elevated globotriaosylsphingosine is a hallmark of Fabry disease. Proc Natl Acad Sci USA. 2008; 105:2812-2817.

45. Chien YH, Lee NC, Chiang SC, Desnick RJ, Hwu WL. Fabry disease: incidence of the common later-onset alpha-galactosidase 
A IVS4+919G $\longrightarrow$ A mutation in Taiwanese newborns superiority of DNA-based to enzyme-based newborn screening for common mutations. Mol Med. 2012; 18:780-784.

46. Ishii S, Nakao S, Minamikawa-Tachino R, Desnick RJ, Fan JQ. Alternative splicing in the alpha-galactosidase A gene: increased exon inclusion results in the Fabry cardiac phenotype. Am J Hum Genet. 2002; 70:994-1002.

47. Hwu WL, Chien YH, Lee NC, Chiang SC, Dobrovolny R, Huang AC, Yeh HY, Chao MC, Lin SJ, Kitagawa T, Desnick RJ, Hsu LW. Newborn screening for Fabry disease in Taiwan reveals a high incidence of the later-onset GLA mutation c. $936+919$ G $>$ A (IVS4+919G $>$ A). Hum Mutat. 2009; 30:1397-1405.

48. Ohshima T, Murray GJ, Swaim WD, Longenecker G, Quirk JM, Cardarelli CO, Sugimoto Y, Pastan I, Gottesman MM, Brady RO, Kulkarni AB. alphaGalactosidase A deficient mice: a model of Fabry disease. Proc Natl Acad Sci USA. 1997; 94:2540-2544.

49. Dinarello CA, Novick D, Kim S, Kaplanski G. Interleukin-18 and IL-18 binding protein. Front Immunol 2013; 4:289.

50. Raeburn CD, Dinarello CA, Zimmerman MA, Calkins CM, Pomerantz BJ, McIntyre RC, Jr., Harken AH, Meng X. Neutralization of IL-18 attenuates lipopolysaccharideinduced myocardial dysfunction. Am J Physiol Heart Circ Physiol. 2002; 283:H650-657.

51. Kahlenberg JM, Thacker SG, Berthier CC, Cohen CD, Kretzler M, Kaplan MJ. Inflammasome activation of IL-18 results in endothelial progenitor cell dysfunction in systemic lupus erythematosus. J Immunol. 2011; 187:6143-6156.

52. Maffia P, Grassia G, Di Meglio P, Carnuccio R, Berrino L, Garside P, Ianaro A, Ialenti A. Neutralization of interleukin-18 inhibits neointimal formation in a rat model of vascular injury. Circulation. 2006; 114:430-437.

53. Gu H, Xie M, Xu L, Zheng X, Yang Y, Lv X. The protective role of interleukin-18 binding protein in a murine model of cardiac ischemia/reperfusion injury. Transpl Int. 2015; 28:1436-1444.

54. Hillestad V, Espe EK, Cero F, Larsen KO, Sjaastad I, Nygard S, Skjonsberg OH, Christensen G. IL-18 neutralization during alveolar hypoxia improves left ventricular diastolic function in mice. Acta Physiol (Oxf). 2015; 213:492-504.

55. Germain DP, Waldek S, Banikazemi M, Bushinsky DA, Charrow J, Desnick RJ, Lee P, Loew T, Vedder AC, Abichandani R, Wilcox WR, Guffon N. Sustained, longterm renal stabilization after 54 months of agalsidase beta therapy in patients with Fabry disease. J Am Soc Nephrol 2007; 18:1547-1557.

56. Thurberg BL, Fallon JT, Mitchell R, Aretz T, Gordon RE, O'Callaghan MW. Cardiac microvascular pathology in Fabry disease: evaluation of endomyocardial biopsies before and after enzyme replacement therapy. Circulation 2009; 119:2561-2567.

57. Keslova-Veselikova J, Hulkova H, Dobrovolny R, Asfaw B, Poupetova H, Berna L, Sikora J, Golan L, Ledvinova J, Elleder M. Replacement of alpha-galactosidase A in Fabry disease: effect on fibroblast cultures compared with biopsied tissues of treated patients. Virchows Arch. 2008; 452:651-665.

58. Chevrier M, Brakch N, Celine L, Genty D, Ramdani Y, Moll S, Djavaheri-Mergny M, Brasse-Lagnel C, Annie Laquerriere AL, Barbey F, Bekri S. Autophagosome maturation is impaired in Fabry disease. Autophagy 2010; 6:589-599.

59. Kampmann C, Linhart A, Baehner F, Palecek T, Wiethoff CM, Miebach E, Whybra C, Gal A, Bultas J, Beck M. Onset and progression of the Anderson-Fabry disease related cardiomyopathy. Int J Cardiol. 2008; 130:367-373. 